University of Nebraska - Lincoln

DigitalCommons@University of Nebraska - Lincoln

$10-2006$

\title{
Efficient Low Dissipative High Order Schemes for Multiscale MHD Flows, II: Minimization of $\mathrm{V} \cdot \mathrm{B}$ Numerical Error
}

Helen C. Yee

NASA Ames Research Center, yee@nas.nasa.gov

Bjorn Sjögreen

Royal Institute of Technology, bjorns@nada.kth.se

Follow this and additional works at: http://digitalcommons.unl.edu/nasapub

Yee, Helen C. and Sjögreen, Bjorn, "Efficient Low Dissipative High Order Schemes for Multiscale MHD Flows, II: Minimization of ७.B Numerical Error" (2006). NASA Publications. 267.

http://digitalcommons.unl.edu/nasapub/267

This Article is brought to you for free and open access by the National Aeronautics and Space Administration at DigitalCommons@University of Nebraska - Lincoln. It has been accepted for inclusion in NASA Publications by an authorized administrator of DigitalCommons@University of Nebraska - Lincoln. 


\title{
Efficient Low Dissipative High Order Schemes for Multiscale MHD Flows, II: Minimization of $\nabla \cdot \mathrm{B}$ Numerical Error*
}

\author{
H. C. Yee $^{1}$ and Björn Sjögreen ${ }^{2}$
}

Received July 22, 2004; accepted (in revised form) April 18, 2005; Published online November 17, 2005

\begin{abstract}
An adaptive numerical dissipation control in a class of high order filter methods for compressible MHD equations is systematically discussed. The filter schemes consist of a divergence-free preserving high order spatial base scheme with a filter approach which can be divergence-free preserving depending on the type of filter operator being used, the method of applying the filter step, and the type of flow problem to be considered. Some of these filter variants provide a natural and efficient way for the minimization of the divergence of the magnetic field $(\nabla \cdot \mathbf{B})$ numerical error in the sense that commonly used divergence cleaning is not required. Numerical experiments presented emphasize the performance of the $\nabla \cdot \mathbf{B}$ numerical error. Many levels of grid refinement and detailed comparison of the filter methods with several commonly used compressible MHD shock-capturing schemes will be illustrated.
\end{abstract}

KEY WORDS: Magnetohydrodynamics; difference scheme; high order of accuracy; shock capturing; numerical divergence.

\section{PRELIMINARIES}

An integrated approach for the control of numerical dissipation in high order finite difference filter schemes in structured curvilinear grids for the compressible Euler and Navier-Stokes equations has been developed

\footnotetext{
*A condensed version appears in the Proceedings of the International Conference on High Performance Scientific Computing, March 10-14, 2003, Hanoi, Vietnam. This is a revised version of a longer internal report, Feb. 19, 2004. The longer internal report was published as a RIACS Technical Report TR03.10, July 2003, NASA Ames Research Center.

${ }^{1}$ NASA Ames Research Center, Moffett Field, California, USA. E-mail: hmcyee@mail.arc. nasa.gov

${ }^{2}$ Royal Institute of Technology, Stockholm, Sweden. E-mail: bjorns@nada.kth.se
} 
and verified by the authors and collaborators [22-25, 32-34]. These filter schemes are suitable for complex multiscale compressible viscous flows, especially for high speed turbulence combustion and acoustics problems. Standard high-resolution shock-capturing schemes are too dissipative for the simulation of these types of flow. Basically, the filter scheme consists of sixth-order or higher spatially centered difference operators as the base scheme. To control the amount and types of numerical dissipation, an artificial compression method (ACM) indicator or multiresolution wavelets are used as sensors to adaptively limit the amount and to aid in the selection and/or blending of the appropriate types of numerical dissipation to be used. This adaptive control of numerical dissipation is accomplished by a filter step after the completion of each full-time step integration of the base scheme. Hereafter, we refer to these schemes as the high order ACM-filter and WAV-filter methods. The following provides the relevant background in extending these filter schemes to the multi-D compressible MHD equations.

This paper is concerned with the compressible MHD equations, which for ease of reference will henceforth be referred to simply as MHD equations. Presently, there are basically two camps in solving the MHD equations; namely, that which solves the conservative form, and that which solves the non-conservative symmetrizable form [11, 19, 20]. For both forms of the MHD equations, high-resolution shock-capturing methods suffer from the need to perform extra work to minimize the $\nabla \cdot \mathbf{B}$ numerical error. The popular procedures for minimizing the $\nabla \cdot \mathbf{B}$ numerical error include augmenting an extra PDE to the system [4], using variants of the staggered grid approach of Yee (including variants of the constraint transport methods) $[5,7,8,39]$ and using variants of a projection method (see e.g. [40]). For ease of reference, hereafter, these existing procedures will be referred to as "standard" or "commonly used" divergence cleanings.

There is a key advantage to solving the conservative equations over the non-conservative symmetrizable equations, since solving the conservative form by a conservative entropy satisfying scheme guarantees correct propagation speeds and locations of discontinuities. The disadvantage is that the conservative form is a non-strictly hyperbolic system with nonconvex inviscid fluxes. There exist states (e.g., triple umbilic points for 1-D) for which the Jacobian of the flux of the conservative form does not have a complete set of eigenvectors, especially for higher than 1-D.

We formulate our filter scheme together with the Cargo and Gallice [3] and Gallice [9,10] form of the multi-D symmetrizable MHD Roe-type approximate Riemann solver for both the conservative and symmetrizable MHD equations. Their Roe-type MHD approximate Riemann solver is an improvement over the Brio and $\mathrm{Wu}$ [2] and Powell [9] approximate 
Riemann solver. A novel feature of our new method introduced in Sjögreen and Yee [26] is that the well-conditioned eigen-decomposition of the symmetrizable MHD equations, with a minor modification (see the next section), is used to solve the conservative equations. This new feature of the method provides well-conditioned eigenvectors for the conservative formulation, so that correct wave speeds for discontinuities are assured by conservative entropy satisfying schemes. It was shown in [26] and will also be shown here that this approach, using the symmetrizable eigensystem when solving the conservative equations, also works well in the context of standard shock-capturing schemes involving the use of the eigen-structure of the MHD equations.

Outline: the present paper is Part II of a series of papers on the subject. This is an expanded version of [35]. Part I [26] introduced some of the basic idea of our new scheme with preliminary study. The present paper present a comprehensive description of the schemes so that interested readers will be able to implement them for practical applications without having to study many additional references. Extensive grid convergence comparisons of these filters schemes with three standard shock-capturing scheme will be conducted in detail. Numerical examples presented emphasize the minimization of the $\nabla \cdot \mathbf{B}$ numerical error. Throughout the paper, the term " $\nabla \cdot \mathbf{B}$ numerical error" refers to the "amount of non-zero value of the discretized form of $\nabla \cdot \mathbf{B}$ of the underlying scheme." The following discussion pertains to schemes involving the use of Riemann solvers or the eigen-structure of the MHD equations. In addition, our discussion is restricted to the finite difference formulation.

\section{A NEW METHOD IN SOLVING THE MULTI-D CONSERVATIVE MHD SYSTEMS}

A full description of our adaptive low dissipative high order filter scheme for the Euler and Navier-Stokes equations can be found in [22, 25, 32-34]. Here, we describe the extension of this scheme to the MHD equations with the blending of high order non-linear filters and high order linear filters for both the conservative and non-conservative symmetrizable systems. An important ingredient in our method is the use of the dissipative portion of high-resolution shock-capturing schemes as part of the non-linear filters. These non-linear filters involve the use of approximate Riemann solvers. A new form of high-resolution shock-capturing schemes for the conservative MHD equations using the non-conservative symmetrizable eigensystem will be elaborated. 


\subsection{Conservative and Symmetrizable Compressible MHD Equations}

The conservative MHD equations are a system of non-strictly hyperbolic conservation laws. It has previously been shown by Powell [19] and Powell et al. [20] that an "almost" equivalent MHD system in non-conservative (symmetrizable) form can be derived. In order to have a better conditioned eigensystem for the application of high-resolution shock-capturing schemes, they adjoined ad hoc non-conservative terms to the conservative equation. The non-conservative symmetrizable form was systematically derived by Godunov [11] 24 years earlier. In 1996, Vinokur showed that the MHD equations can be derived from basic principles in either conservative or non-conservative symmetrizable form [29].

Consider the 3-D conservative and non-conservative symmetrizable forms of the ideal compressible MHD equations in Cartesian grids,

$$
\begin{gathered}
\left(\begin{array}{c}
\rho \\
\rho u \\
\rho v \\
\rho w \\
e \\
B_{x} \\
B_{y} \\
B_{z}
\end{array}\right)_{t}+\operatorname{div}\left(\begin{array}{c}
\rho \mathbf{u} \\
\rho \mathbf{u u}^{T}+\left(p+B^{2} / 2\right) I-\mathbf{B B}^{T} \\
\mathbf{u}\left(e+p+B^{2} / 2\right)-\mathbf{B}\left(\mathbf{u}^{T} \mathbf{B}\right) \\
\mathbf{u B}^{T}-\mathbf{B} \mathbf{u}^{T}
\end{array}\right) \\
\left(\begin{array}{c}
\rho \\
\rho u \\
\rho v \\
\rho w \\
e \\
B_{x} \\
B_{y} \\
B_{z}
\end{array}\right)_{t}+\operatorname{div}\left(\begin{array}{c}
\rho \mathbf{u u}^{T}+\left(p+B^{2} / 2\right) I-\mathbf{B} \mathbf{B}^{T} \\
\mathbf{u}\left(e+p+B^{2} / 2\right)-\mathbf{B}\left(\mathbf{u}^{T} \mathbf{B}\right) \\
\mathbf{u B} \mathbf{B}^{T}-\mathbf{B} \mathbf{u}^{T}
\end{array}\right)=-\left(\begin{array}{c}
0 \\
0 \\
B_{x} \\
B_{y} \\
B_{z} \\
\mathbf{u}^{T} \mathbf{B} \\
u \\
v \\
w
\end{array}\right) \\
\text { (symmetrizable), }
\end{gathered}
$$

where the velocity vector $\mathbf{u}=(u, v, w)^{T}$, the magnetic field vector $\mathbf{B}=$ $\left(B_{x}, B_{y}, B_{z}\right)^{T}, \rho$ is the density, and $e$ is the total energy. The notation $B^{2}=$ $B_{x}^{2}+B_{y}^{2}+B_{z}^{2}$ is used. The pressure is related to the other variables by

$$
p=(\gamma-1)\left(e-\frac{1}{2} \rho\left(u^{2}+v^{2}+w^{2}\right)-\frac{1}{2}\left(B_{x}^{2}+B_{y}^{2}+B_{z}^{2}\right)\right) .
$$

For plasmas, $\gamma$ is usually equal to $5 / 3$ (for monatomic gases). 
The vector on the right-hand side of (2) is the non-conservative portion of the symmetrizable MHD system, which is frequently referred to in the literature as a source term vector. The authors prefer not to use this nomenclature since this is part of the symmetrizable form of the MHD and it is not a source term. That is, the symmetrizable form of the MHD (2) is written in two parts; namely, a conservative portion and a non-conservative portion. The non-conservative portion is proportional to $\nabla \cdot \mathbf{B}$. Physically, it is zero if $\nabla \cdot \mathbf{B}=0$ initially. Hereafter, the terms "symmetrizable system (or non-conservative symmetrizable)" and "non-conservative system" are used interchangeably.

In symbolic form the conservative and non-conservative forms can be written as

$$
\begin{aligned}
& U_{t}+\nabla \cdot \mathbf{F}=0, \\
& U_{t}+\nabla \cdot \mathbf{F}=S,
\end{aligned}
$$

respectively, where $U$ is the corresponding state vector, $\mathbf{F}$ is the conservative inviscid flux vector tensor and $S$ is the non-conservative portion of the equations in (2).

\subsection{Solving the Conservative and Symmetrizable Systems Involving the Use of Approximate Riemann Solvers}

For convenience of presentation we will describe our numerical methods for the $x$-flux on a uniform grid. The schemes to be discussed, in most part, only spell out the $x$-component terms with the $y$ - and $z$-components omitted. Let $A(U)$ denote the Jacobian $\partial F / \partial U$ with the understanding that the present $F$ and $S$ are the $x$-component of the 3-D description above. For later discussion we write the non-conservative term $S$ in the $x$-direction as $N(U) U_{x}$.

Gallice [9] and Cargo and Gallice [3] made use of the fact that seven of the eigenvalues and eigenvectors are identical for the "conservative" Jacobian matrix $A$ and the "non-conservative" Jacobian matrix $(A-N)$. For ease of reference, we refer to the distinct eigenvalue (eigenvector) between the conservative and non-conservative MHD as the eighth eigenvalue (eigenvector). The eighth eigenvector of $A$ of the conservative system associated with the degenerate zero eigenvalue can sometimes coincide with one of the other eigenvectors, thereby, making it difficult to obtain the Roe-type approximate Riemann solver for the multi-dimensional conservative MHD. On the other hand, the eigenvectors of the nonconservative Jacobian $A^{*}=(A-N)$ always form a complete basis, and 
can be obtained from analytical formulas [11, 19] for 1-D or higher. A Roe-type average state was developed in [3] for 1-D conservative MHD and extended to the 3-D non-conservative MHD in [9, 10]. Their form is an improvement over the Brio and $\mathrm{Wu}$ [2] and Powell [19] average state.

The construction of the Gallice Roe-type average state for the conservative system (with $F$ and $A$, the flux function and the Jacobian in the $x$ direction, respectively) satisfying the following

$$
F\left(U_{R}, U_{L}\right)=A\left(U_{R}, U_{L}\right)\left(U_{R}-U_{L}\right)=\bar{A}\left(U_{R}-U_{L}\right)
$$

does not satisfy the specific Roe's average state perfect gas dynamic condition. For a perfect gas in gas dynamics, there exists a Roe average state $\bar{U}$ such that (assuming $A^{\mathrm{pg}}$ as the Jacobian of the inviscid gas dynamic flux)

$$
A^{\mathrm{pg}}\left(U_{R}, U_{L}\right)=A^{\mathrm{pg}}(\bar{U}) .
$$

For the non-conservative MHD system, it is not possible to obtain the MHD equivalent of $A^{\mathrm{pg}}(\bar{U})$ for $\bar{A}$ due to the $\mathbf{B}^{2}$ term. Gallice's average state is a combination of Roe-type average state for certain MHD flow variables, a mean average state and a new average state for $\mathbf{B}$. We formulate our scheme together with the Gallice form of the 3-D non-conservative MHD Roe-type approximate Riemann solver for both the conservative and non-conservative MHD equations.

\subsection{Solving the Conservative System Using the Symmetrizable Eigenvectors}

We propose to use eigenvectors of the non-conservative form but with the degenerate eigenvalue replaced by an entropy correction [12, 31] of what was supposed to be the zero eigenvalue (e.g., a small parameter $\epsilon$ that is scaled by the largest eigenvalue of $A(U)$ ) for the conservative form. In the present context, the use of the non-zero entropy correction is different from the standard entropy violation associated with expansion shocks in the Roe-type approximate solver in gas dynamics, since the conservative inviscid gas dynamics equations are strictly hyperbolic. For more than one-space dimension, a multi-dimension entropy correction as proposed in [31] is used for each of the degenerate eigenvalues in each spatial direction. Our rationale for doing this is that only the eighth eigenvector of the nonconservative form is not the same as the eighth eigenvector for the conservative form. The incorrect eigenvector for the conservative form will be multiplied by an eigenvalue which is close to zero (the eigenvalue will not be exactly zero when an entropy correction is used). Thus the effect of a "false" eigenvector will be small. By using the eighth eigenvector of the 
non-conservative system instead, the difficulty of dealing with an incomplete set of eigenvectors for the conservative system can be avoided.

From here on, the use of ACM-filter, WAV-filter, Harten-Yee, MUSCL or the fifth-order WENO [13] (WENO5) scheme in solving the conservative MHD (1) in this paper means the used of the non-conservative eigen-decomposition described above in solving the conservative MHD equation set (1), whereas the corresponding schemes solving the symmetrizable equation set (2) means the used of its own non-conservative eigen-decomposition.

\section{DESCRIPTION OF HIGH ORDER FILTER METHODS}

Our high order ACM-filter and WAV-filter methods consist of two steps, a base scheme step (not involving the use of approximate Riemann solvers or flux limiters) and a filter step (involving the use of approximate Riemann solvers and flux limiters). The filter step can be divergence-free preserving depending on the type of filter operator being used and the method of applying the filter step. In order to have good shock-capturing capability and improved non-linear stability related to spurious high frequency oscillations, the blending of a high order non-linear filter and a high order linear filter was proposed in our gas dynamic schemes [34]. The non-linear filter consists of the product of an ACM or wavelet sensor and the non-linear dissipative portion of a high-resolution shock-capturing scheme. The high order linear filter consists of the product of another sensor, a tuning parameter and a high order centered linear dissipative operator that is compatible with the order of the base scheme being used. Here the extension with a modification of the gas dynamic filter approach to the MHD equations that minimizes the $\nabla \cdot \mathbf{B}$ numerical error is discussed.

\subsection{Divergence-Free Preserving Base Scheme Step}

The first step of the numerical method consists of a time step by a high order non-dissipative spatial and high order temporal base scheme operator $L^{*}$ (e.g., a sixth-order central in space and high order linearmultistep or fourth-order Runge-Kutta in time). After the completion of a full-time step of the base scheme step, the solution is denoted by $U^{*}$

$$
U^{*}=L^{*}\left(U^{n}\right)
$$

where $U^{n}$ is the numerical solution vector at time level $n$. Note that for more than two time level linear-multistep methods (LMMs) as time discretizations, the $L^{*}$ operator involves the corresponding number of time 
levels. For higher than first-order Runge-Kutta method, the $L^{*}$ operator involves the corresponding number of stages of spatial discretization of the flux derivatives.

When necessary, a high order linear numerical dissipation operator can be added to the base scheme. For example, an eighth-order linear dissipation with the sixth-order centered base scheme to approximate $F(U)_{x}$ (with the grid indices $k$ and $l$ for the $y$-and $z$-directions suppressed) is written as

$$
\frac{\partial F}{\partial x} \approx D_{06} F_{j}+d(\Delta x)^{7}\left(D_{+} D_{-}\right)^{4} U_{j},
$$

where $D_{06}$ is the standard sixth-order accurate centered difference operator, and $D_{+} D_{-}$is the standard second-order accurate centered approximation of the second derivative. The small parameter $d$ is a scaled value in the range of $0.00001-0.005$, depending on the flow problem, and has the sign which gives dissipation in the forward time direction. The $D_{06}$ operators is modified at boundaries in a stable way by the so-called summation-by-parts (SBP) operators [14-17, 34]. See the end of this section for a discussion. The linear numerical dissipation operator $D_{+} D_{-}$is modified at the boundaries to be semi-bounded [21].

For example, the base scheme step with the fourth-order classical Runge-Kutta time discretization takes the form

$$
\begin{aligned}
& k_{1}=L\left(U^{n}\right), \\
& k_{2}=L\left(U^{n}+\frac{\Delta t}{2} k_{1}\right), \\
& k_{3}=L\left(U^{n}+\frac{\Delta t}{2} k_{2}\right), \\
& k_{4}=L\left(U^{n}+\Delta t k_{3}\right), \\
& U^{*}=U^{n}+\frac{\Delta t}{6}\left[k_{1}+2 k_{2}+2 k_{3}+k_{4}\right],
\end{aligned}
$$

where the $L$ operator is a semi-discrete form of the conservative system (1) or non-conservative system (2). For example, using formula (4) to discretize (1) with the $\mathbf{F}=(F, G, H)$ flux vector tensor takes the form

$$
\begin{aligned}
\frac{d\left(U_{j, k, l}\right)}{d t}= & L(U)_{j, k, l} \\
= & -\left\{\left(D_{j}\right)_{06} F_{j, k, l}+\left(d_{x}\right)(\Delta x)^{7}\left[\left(D_{j}\right)_{+}\left(D_{j}\right)_{-}\right]^{4} U_{j, k, l}\right. \\
& +\left(D_{k}\right)_{06} G_{j, k, l}+\left(d_{y}\right)(\Delta y)^{7}\left[\left(D_{k}\right)_{+}\left(D_{k}\right)_{-}\right]^{4} U_{j, k, l} \\
& \left.+\left(D_{l}\right)_{06} H_{j, k, l}+\left(d_{z}\right)(\Delta z)^{7}\left[\left(D_{l}\right)_{+}\left(D_{l}\right)_{-}\right]^{4} U_{j, k, l}\right\},
\end{aligned}
$$

where $D_{j}, D_{k}$, and $D_{l}$ denote finite difference operators acting in the $j$-, $k$-, $l$-directions, respectively. Here for example $\left(D_{j}\right)_{06}$ denotes the sixthorder centered difference operator in the $x$-direction. Similarly, $\left(D_{j}\right)_{+}\left(D_{j}\right)_{-}$ 
denotes the second-order centered approximation of the second derivative in the $j$-direction. The small parameters $d_{x}, d_{y}$, and $d_{z}$ are the same as $d$ in (4). The base scheme operator $L^{*}$ in (3) in conjunction with the fourthorder Runge-Kutta (5) and (6) becomes

$$
U^{*}=L^{*}\left(U^{n}\right)=U^{n}+\frac{\Delta t}{6}\left[k_{1}+2 k_{2}+2 k_{3}+k_{4}\right] .
$$

This highly accurate spatial base scheme is employed to numerically preserve the divergence-free condition of the magnetic field (to the level of round-off error) for uniform Cartesian grids with periodic boundary conditions. For example, when using pure centered difference operators, it is trivial to see that the divergence of $\mathbf{B}$ is perfectly preserved. Take, for example, the semi-discrete approximation of the magnetic field equations

$$
\begin{aligned}
\frac{d B_{x}(t)_{j, k, l}}{d t} & + \\
& D_{k}\left[\left(B_{y}\right)_{j, k, l} u_{j, k, l}-\left(B_{x}\right)_{j, k, l} v_{j, k, l}\right]+D_{l}\left[\left(B_{z}\right)_{j, k, l} u_{j, k, l}-\left(B_{x}\right)_{j, k, l} w_{j, k, l}\right]=0, \\
\frac{d B_{y}(t)_{j, k, l}}{d t} & + \\
& D_{j}\left[\left(B_{x}\right)_{j, k, l} v_{j, k, l}-\left(B_{y}\right)_{j, k, l} u_{j, k, l}\right)+D_{l}\left(\left(B_{z}\right)_{j, k, l} v_{j, k, l}-\left(B_{y}\right)_{j, k, l} w_{j, k, l}\right]=0, \\
\frac{d B_{z}(t)_{j, k, l}}{d t} & + \\
& D_{j}\left[\left(B_{x}\right)_{j, k, l} w_{j, k, l}-\left(B_{z}\right)_{j, k, l} u_{j, k, l}\right]+D_{k}\left[\left(B_{y}\right)_{j, k, l} w_{j, k, l}-\left(B_{z}\right)_{j, k, l} v_{j, k, l}\right]=0,
\end{aligned}
$$

where $D_{j}, D_{k}$, and $D_{l}$ here denote finite difference centered operators acting in the $j$-, $k$-, $l$-directions, respectively.

Forming the divergence by taking the sum of $D_{j}$ on the $B_{x}$ equation, $D_{k}$ on the $B_{y}$ equation, and $D_{l}$ on the $B_{z}$ equation gives

$$
\frac{d\left(D_{j} B_{x}+D_{k} B_{y}+D_{l} B_{z}\right)_{j, k, l}}{d t}=-D_{j} D_{k}\left[\left(B_{y}\right)_{j, k, l} u_{j, k, l}\right]+D_{k} D_{j}\left[\left(B_{y}\right)_{j, k, l} u_{j, k, l}\right]+\cdots=0,
$$

where the dots denote several additional terms of a form similar to the first two. All these terms disappear, since the difference operators along different coordinate directions commute, i.e., $D_{j} D_{k}=D_{k} D_{j}$. Similarly, $\nabla \cdot \mathbf{B}$ is perfectly preserved for the base scheme that includes a high order linear dissipation term (4) applied to the magnetic field equations. The same property of the base scheme holds for the non-conservative system (2).

When the solution is smooth, the filter step might not be needed. Thus the use of a high order centered difference operator as the base scheme will perfectly preserve the divergence-free condition (for periodic boundary conditions and open boundaries without complex wave structure near the physical boundaries). In this case the result will be the same, whether we solve the conservative system (1) or non-conservative system (2). It is noted that for open boundaries with complex wave interactions near the physical boundaries, it is not absolutely clear how different it is 
in solving system (1) and system (2) for the base scheme step. The study on this issue is forthcoming. We discuss below numerical boundary treatment for high order methods for non-periodic physical boundaries.

Stable Numerical Boundary Treatments in Spatially High Order Centered Base Schemes versus. Divergence Free Preserving: the type of spatially high order centered base schemes used in the ACM-filter and WAV-filter methods [22, 25, 32-34] is divergence-free preserving for the multi-dimensional MHD equations for periodic boundary conditions and for open boundaries without complex wave structures near the physical boundaries. Even for these types of physical boundary conditions, straightforward application of the filter step to the MHD equations will not automatically preserve the divergence-free magnetic field condition numerically since the filter step involves the non-linear dissipative portion of standard shock-capturing schemes. On the other hand, with careful modification of the gas dynamics scheme, the filter mechanism offers several natural and efficient alternatives (without the commonly used divergence cleaning procedures) for minimizing the $\nabla \cdot \mathbf{B}$ numerical error. For ease of reference, divergence-free base schemes for the interior points (away from the computational boundaries), for periodic boundary conditions, and for open boundaries without complex wave structure near the physical boundaries, will be henceforth referred to as "divergence-free preserving base schemes".

For non-periodic boundary conditions, the stable numerical boundary condition treatments for spatially high order methods for non-linear systems of hyperbolic conservation laws are not straightforward, unlike their second-order or lower schemes. For higher than second-order centered schemes, a discrete $L^{2}$ energy norm stability (SBP stable numerical boundary conditions) provides the required numerical boundary schemes [14-17]. The SBP type of stable numerical boundary conditions is employed in this paper. It is important to point out that the requirement for a spatially stable high order centered scheme (including boundary schemes) is potentially conflicting with the desire to be divergence-free preserving for general multi-dimensional open boundaries. These stable $L^{2}$ energy norm conditions impose non-traditional numerical boundary treatments and are not divergence-free preserving in a general multi-dimensional open boundary setting, unless the open boundaries are very smooth in all spatial directions with the computational domain large enough to not be affected by the reflecting waves at the boundaries. The grid stencil of these SBP boundary operators is very wide. Lowering the order of the centered scheme near the open boundaries, a non-reflecting boundary condition treatment, and/or enlargement of the computational domain if appropriate might help to minimize the $\nabla \cdot \mathbf{B}$ numerical error. Research on 
divergence-free preserving stable numerical boundary conditions for open physical boundaries for high order centered schemes is ongoing.

\subsection{Adaptive Numerical Dissipation Filter Step}

After the completion of a full-time step of the divergence-free preserving base scheme step, the second step is to adaptively filter the solution by the product of "an ACM indicator or wavelet sensor" and the "non-linear dissipative portion of a high-resolution shock-capturing scheme." If necessary, the blending of a high order linear filter with a non-linear filter [34] will be used. The final update step after the filter step can be written (with some of grid indices suppressed for ease of illustration) as

$$
\begin{aligned}
U_{j, k, l}^{n+1}=U_{j, k, l}^{*} & -\frac{\Delta t}{\Delta x}\left[H_{j+1 / 2}^{f x}-H_{j-1 / 2}^{f x}\right]-\frac{\Delta t}{\Delta y}\left[H_{k+1 / 2}^{f y}-H_{k-1 / 2}^{f y}\right] \\
& -\frac{\Delta t}{\Delta z}\left[H_{l+1 / 2}^{f z}-H_{l-1 / 2}^{f z}\right] .
\end{aligned}
$$

Here, $H_{j \pm 1 / 2}^{f x}, H_{k \pm 1 / 2}^{f y}$, and $H_{l \pm 1 / 2}^{f z}$ are the filter numerical fluxes in the $x, y$, and $z$ directions, respectively. The $x$-filter numerical flux vector $H_{j+1 / 2}^{f x}$ is

$$
H_{j+1 / 2}^{f x}=R_{j+1 / 2} \bar{H}_{j+1 / 2},
$$

where $R_{j+1 / 2}$ is the matrix of right eigenvectors of the Jacobian of the non-conservative MHD flux vector $\left(A_{j+1 / 2}-N_{j+1 / 2}\right)$ evaluated at the Gallice average state in terms of the $U^{*}$ solution from the base scheme step (3). The notation, for example $R_{j+1 / 2}$ stands for $R_{j+1 / 2, k, l}$ and the subscript in $R_{j+1 / 2}$ indicates the average state evaluated in the $x$-direction of the eigenvectors in terms of $U^{*}$. See $[10,27]$ or Appendix A for the average state formula for the 3 -D non-conservative system (2). The $\bar{H}_{j+1 / 2}$ (involving the use of flux limiters) are also evaluated from the same average state. The dimension-by-dimension procedure of applying the approximate Riemann solver is adopted.

\subsection{Blending of a Non-Linear Filter with a High Order Linear Filter}

Option I: "filter all" a straightforward extension of our gas dynamic filter scheme for the MHD equations using the blending of a non-linear filter with a high order linear filter to all equations of (1) or (2) take the following form. Denote the elements of the vector $\bar{H}_{j+1 / 2}$ by $\bar{h}_{j+1 / 2}^{l}, l=$ 
$1,2, \ldots, 8$. They have the form

$$
\bar{h}_{j+1 / 2}^{l}=\frac{1}{2}\left(s^{N}\right)_{j+1 / 2}^{l}\left(\phi_{j+1 / 2}^{l}\right)-\left(s^{L}\right)_{j+1 / 2}^{l} d_{j+1 / 2}^{l} .
$$

Here $\left(s^{N}\right)_{j+1 / 2}^{l}$ and $\left(s^{L}\right)_{j+1 / 2}^{l}$ are sensors to activate the higher order non-linear filter and linear filter, respectively. For example, $\left(s^{N}\right)_{j+1 / 2}^{l}$ is designed to be zero or near zero in regions of smooth flow and near one in regions with discontinuities. $\left(s^{N}\right)_{j+1 / 2}^{l}$ varies from one grid point to another and is obtained either from a wavelet analysis of the flow solution (WAV-filter scheme), or from a gradient-based detector (ACM-filter scheme) [22, 25, 32-34]. We have in the previous numerical experiments [34] used $\left(s^{L}\right)_{j+1 / 2}^{l}=1-\left(s^{N}\right)_{j+1 / 2}^{l}$, but other choices are possible (see [34] or Appendix B for the sensor formula). The functions $\phi_{j+1 / 2}^{l}$ and $d_{j+1 / 2}^{l}$ are the dissipative portion of the respective non-linear and linear filters for the local $l$ th-characteristic wave [34] in the $x$-direction.

Note that the wavelet sensor can be obtained from the characteristic variables for each wave or a single sensor for all eight waves, based on pressure and density. Both methods were implemented but, for the numerical test in this paper, the simpler non-characteristic sensor was employed.

The dissipative portion of the non-linear filter $\phi_{j+1 / 2}^{l}=g_{j+1 / 2}^{l}-b_{j+1 / 2}^{l}$ is the dissipative portion of a high order high-resolution shock-capturing scheme for the local $l$ th-characteristic wave. Here $g_{j+1 / 2}^{l}$ and $b_{j+1 / 2}^{l}$ are numerical fluxes of the uniformly high order high-resolution scheme and a high order central scheme for the $l$ th-characteristic, respectively. It is noted that $b_{j+1 / 2}^{l}$ might not be unique since there is more than one way of obtaining $\phi_{j+1 / 2}^{l}$. For the forms of the $\phi_{j+1 / 2}^{l}$ used in the numerical experiment section, (see[22, 25, 32-34] or Appendix B for a description). For example, the form of Harten and Yee and symmetric TVD schemes are already in the proper form in the sense that they are written in a central differencing portion $b_{j+1 / 2}^{l}$ and a non-linear dissipation portion $\phi_{j+1 / 2}^{l}$. No work is required to obtain $\phi_{j+1 / 2}^{l}$ in this case. A comparison of using three different dissipative portions of typical shock-capturing schemes (Harten-Yee, MUSCL and WENO5) is reported in [38].

For the dissipative portion of the linear filter in the second term of eight, for example an eighth-order linear filter in terms of the local characteristic variables has the form

$$
d_{j+1 / 2}^{l}=\frac{1}{2} d_{f} \Delta x^{6} D_{+}\left(D_{+} D_{-}\right)^{3}\left(w_{j+1 / 2}^{l}+w_{j-1 / 2}^{l}\right)
$$

or

$$
d_{j+1 / 2}^{l}=d_{f} \Delta x^{6} D_{+}\left(D_{+} D_{-}\right)^{3} w_{j}^{l} .
$$


Here $w_{j}^{l}$ is the local $l$ th-characteristic variable in the $x$-direction evaluated at $U^{*}$. The term $w_{j+1 / 2}^{l}$ is the local $l$ th-characteristic variable in the $x$-direction evaluated at the average state in terms of $U^{*} . d_{f}$ is a small tuning parameter with a range scaled range as the parameter $d$ in (4). The sign of $d_{f}$ giving dissipation in the forward time direction. For a sixthorder spatial base scheme, the eighth-order central dissipation for the linear filter is used. For the test cases to be shown later, all the computations use only the non-linear filter. i.e., setting $\left(s^{L}\right)_{j+1 / 2}^{l}=0$. Note that the high order linear filter is not to be confused with the high order linear dissipation in the base scheme step (4).

An alternative to applying the linear filter in terms of the characteristic variables [second term in (8)], is to apply it in terms of the conservative variables. In this case, we split

$$
H_{j+1 / 2}^{f x}=R_{j+1 / 2} \bar{H}_{j+1 / 2}=R_{j+1 / 2} \bar{H}_{j+1 / 2}^{N}+\bar{H}_{j+1 / 2}^{L},
$$

where elements of $\bar{H}_{j+1 / 2}^{N}$ are (8) with the second term not present. The linear filter $\bar{H}_{j+1 / 2}^{L}$ now has the form

$$
\bar{H}_{j+1 / 2}^{L}=\frac{1}{2} s^{\text {wav }} d_{f} \Delta x^{6} D_{+}\left(D_{+} D_{-}\right)^{3}\left(U_{j+1 / 2}+U_{j-1 / 2}\right)
$$

or

$$
\bar{H}_{j+1 / 2}^{L}=s^{\text {wav }} d_{f} \Delta x^{6} D_{+}\left(D_{+} D_{-}\right)^{3} U_{j} .
$$

Here $s^{\text {wav }}$ is the wavelet sensor based on the pressure and density at the grid point $j+1 / 2$.

The blended filter given by (8) or the alternative discussed above, if applied to the entire MHD system (denoted by "filter all") will not preserve the divergence free magnetic field condition with the exception of using the WAV-filter scheme for certain smooth flows (see the numerical examples section for an example). This is due to the fact that the WAV-filter scheme sensor turns off the non-linear filter at regions of very smooth flow, whereas the ACM-filter only reduces the strength of the nonlinear filter (see Appendix B or [22] for the comparison of the two sensors). The next subsection describes some alternatives.

\subsection{Additional Options in Filtering the Magnetic Field Equations}

The need to minimize the $\nabla \cdot \mathbf{B}$ numerical error and to achieve a highresolution numerical solution on the inherent flow structure of a particular 
problem are often conflicting. Without special care on the non-linear filter step, the $\nabla \cdot \mathbf{B}$ numerical error might overwhelm the overall accuracy of the magnetic field numerical solutions. Four options were proposed in [36] to minimize the $\nabla \cdot \mathbf{B}$ numerical error over the "filter all" approach. Only one option is discussed here.

Option II: "no filter on B" For this option, the non-linear filter step [for $\left(s^{L}\right)_{j+1 / 2}^{l}=0$ of (8)] only acts on the gas dynamic portion of the equations. That is, the non-linear filter step (8) only applies to the first five equations of (1) or (2). The no filter on the magnetic field equations is denoted by "no filter on B." Here the complete set of eigenvalues and eigenvectors of the full MHD system is used to evaluate the first five equations of (1) or (2). With the divergence free spatial base scheme, the divergence free property should be preserved.

\section{2-D COMPRESSIBLE MHD NUMERICAL EXAMPLES}

A highly parallel 3-D compressible viscous MHD code was built based on our ACM-filter and WAV-filter schemes in curvilinear grids. It was tested on a 3-D curvilinear grid problem. Here, in order to fully test the "No Filter on B" filter approach first, only two-space dimensions are chosen (using the same 3-D code with one of the dimensions not activated) in order to complete the study in a reasonable time frame. This is due largely to the fact that the MHD equations are a larger system of equations than their gas dynamics counterpart.

\subsection{Summary of Numerical Experiments}

Four different 2-D MHD test cases commonly used in the literature were chosen; namely, the Kelvin-Helmholtz, 2-D Riemann, Orszag-Tang vortex and shock/magnetic cloud interaction problems. The first and the third test cases consist of periodic boundary conditions and the other test cases consist of open boundaries. These test cases, mostly shock wave dominated, represent four different flow types which typically arise in computational astrophysics and space weather forecasting. They are chosen to fully validate our proposed schemes. They are not shock/turbulence/combustion problems. The performance of our filter approach for multiscale physical problems will be reported in a forthcoming paper. It is reminded that the full capability of the new scheme is not utilized on these test cases. Thus, we do not expect the new scheme to exhibit drastic improvement in shock-resolution over conventional shock-capturing schemes, since these filter schemes use the same flux limiters to control 
the spurious oscillation across discontinuities as any good high-resolution shock-capturing scheme. Resolution will be gained in regions away from discontinuities on flows with complex structures such as turbulent fluctuations due to the filter approach. See [34] for the performance of the filter approach for long time integration of shock/turbulence interaction gas dynamic flows. In all test cases, the high order linear filter in (8) is not needed for option I. Hereafter, we refer to option I with $\left(s^{L}\right)_{j+1 / 2}^{l}=0$ in (8) as "filter all."

Five shock-capturing schemes, notation and time discretizations: The sixth-order base scheme $[d=0$ in (4)] together with the non-linear/linear filter with wavelet sensor will be denoted WAV66. When a gradient-based sensor ACM is used, the scheme is denoted ACM66. The second number indicates the order of the scheme for discretizing the viscous fluxes, if present. To adhere to the convention of previous work, even though we are dealing with inviscid flows, the same notation is used (see [37, 38] for some non-ideal computations). If high order linear numerical dissipation is also used in the base scheme $[d \neq 0$ in (4)], the methods will be denoted WAV66+AD8 and ACM66+AD8, respectively. The strength of the eighthorder dissipation tunable coefficient $d$ in (4) is in the range of $(0.0001$, 0.01 ). For some of the test cases and flux limiter combinations, $d=0.0001$ exhibits small spurious oscillations due to insufficient dissipation on the base scheme step. For all test cases, $d=0.001$ exhibits less $\nabla \cdot \mathbf{B}$ numerical error than $d=0.01$. Only results for $d=0.001$ will be shown. In all of the filter scheme computations, the non-linear dissipative portion of Harten-Yee is used as part of the non-linear filter term $\phi_{j+1 / 2}^{l}$ (8) (See Appendix B). A comparison of using three different non-linear dissipative portions of typical shock-capturing schemes (Harten-Yee, MUSCL and WENO5) as part of $\phi_{j+1 / 2}^{l}(8)$ is reported in [38]. The resolution of these three different dissipative portions of shock-capturing schemes is comparable with similar efficiency. The entropy fix parameter $\epsilon$ is 0.25 for the Harten-Yee, MUSCL, ACM, and WAV-filter schemes. The cut off wavelet Lipschitz exponent $\beta$ is 0.5 [22] for the WAV-filter scheme. The ACM, parameter $\kappa$ is $0.5,0.7,0.9$ and 0.9 for the four test cases, respectively (see[22, 32, 34] or Appendix B for the definition of $\kappa$ and $\beta$ ).

The fifth-order (WENO5), and second-order Harten-Yee and secondorder MUSCL schemes are used for comparison. Classical fourth-order Runge-Kutta time stepping is used for all sixth-order schemes, as well as for the WENO5 scheme. The second-order Harten-Yee and MUSCL are integrated in time by the second-order TVD Runge-Kutta method. Solving the conservative and non-conservative systems of these three standard shock-capturing schemes as well as the ACM- and WAV-filter schemes is 
considered. All the methods use the same approximate Riemann solver of Gallice in uniform Cartesian grids. For the conservative form of the MHD equations, the non-conservative eigenvector decomposition as discussed in the previous section is employed for the filter step. Unless otherwise indicated on the figures, all results solve the conservative system using the eigenvector of the non-conservative system. When needed, the notation "Cons" denotes computations solving the conservative system and "Non-cons" denotes computations solving the non-conservative system.

Flux limiters: Except for WENO5, the minmod limiter, the van Leer version of the van Albada limiter and the Colella-Woodward limiter are considered (see [31] or Appendix B for the form of limiters being used in the computations). For the second-order MUSCL scheme, the limiter is applied to the primitive variables. In general, all three limiters are stable for the test cases. The van Albada and Colella-Woodward limiters exhibit better accuracy than the minmod limiter when using coarse grids. The minmod limiter is the most dissipative limiter among the three and thus appears to be more stable with smear shock/contact profiles at the expense of requiring a finer grid for the same resolution. In a few isolated cases, the Colella-Woodward limiter exhibits small spurious oscillations whereas the van Albada limiter does not. Due to this fact, unless indicated, all figures use the van Albada limiter. It is noted that a large number of MHD computations reported in the literature employ the minmod limiter due to instability or moderate spurious oscillations encountered in using the other two limiters on their shock-capturing schemes.

For all test cases, except for WENO5, the CFL used is in the range of $(0.4,0.6)$. In some cases, even a higher CFL (up to 0.9) can be used. For WENO5, the CFL used is in the range of $(0.1,0.4)$.

Second-order versus higher-order spatial schemes: For each of the four test cases, in general, the higher-order methods require half the number of grid points in each direction over the second-order methods for coarse grids, except for the 2-D Riemann problem. Since most of the 2-D Riemann test problem consists of no structure away from discontinuities, there is no gain by employing higher-order methods over second-order methods. However, the purpose of choosing this problem is to observe in the simplest way the amount of $\nabla \cdot \mathbf{B}$ numerical error by the different schemes due mainly to discontinuities capturing mechanisms. As the grid is refined, the majority of the methods, if convergent, have resolutions very similar for their gas dynamic solutions. However, the magnetic field variables and the $\nabla \cdot \mathbf{B}$ numerical errors are very different from method to method. In order to give an indication of the the gas dynamic flow structure including all discontinuities for each test case, only representative 
density contours will be shown. The measure of the $\nabla \cdot \mathbf{B}$ numerical error for the various schemes are as follows.

Measure of the $\nabla \cdot \mathbf{B}$ numerical errors: It is well known in computational MHD circles that standard upwind shock-capturing schemes without any divergence cleaning can exhibit large $\nabla \cdot \mathbf{B}$ numerical errors even if the accuracy of their gas dynamic variables is not drastically affected. There is also no common consensus in measuring the $\nabla \cdot \mathbf{B}$ numerical error effectively in the literature when dealing with a large amount of comparison. This is compounded by the fact that the order of the scheme plays a role in how to define the discrete $\nabla \cdot \mathbf{B}$ numerical errors. The authors consider two forms in measuring these errors, namely; (a) examine the discrete $\nabla \cdot \mathbf{B}$ contour at a certain stage of the evolution process and (b) examine the discrete $L^{2}$-norm for the entire time evolution of interest.

The $\nabla \cdot \mathbf{B}$ numerical error is obtained by approximating the $\nabla \cdot \mathbf{B}$ by sixth-order centered differences for WAV66 (WAV66+AD8), ACM66 (ACM66+AD8), and WENO5, whereas the corresponding $\nabla \cdot \mathbf{B}$ numerical error is obtained by second-order centered differences for the second-order TVD schemes (MUSCL and Harten-Yee). The $L^{2}$-norm of $\nabla \cdot \mathbf{B}$ of a particular scheme is computed by taking the square root of the sum over all spatial directions of the square of the discretized form of $\nabla \cdot \mathbf{B}$ at all grid points, including boundary points. These two measures complement each other and give a global picture of the numerical error in $\nabla \cdot \mathbf{B}$ and, indirectly the $\mathbf{B}$ fields. However, these two measures will not illustrate the isolated instances where one of the magnetic field errors is more pronounced than the others. In light of the number of schemes and their variants and the different flow structure of each test case, searching for such isolated instances will not be undertaken.

From the above definitions, the discrete $\nabla \cdot \mathbf{B}$ and discrete $L^{2}$-norm of $\nabla \cdot \mathbf{B}$ are different between the second-order methods and their higher-order counterparts. For each of the test cases, the range and the number of the contours shown are determined by the largest error among the considered methods. Care must be taken when interpreting the results. If one of the methods exhibits a much larger $\nabla \cdot \mathbf{B}$ error than the rest, the small error might appear to be without any contour line on the plot (blank plot). Thus, not all of the blank (empty) $\nabla \cdot \mathbf{B}$ contour plots are an indication of zero $\nabla \cdot \mathbf{B}$ numerical error (machine zero) at that particular time instance. The discrete $L^{2}$-norm of $\nabla \cdot \mathbf{B}$ time history curve value should be used as a check if blank $\nabla \cdot \mathbf{B}$ contours are truly divergence-free preserving at that specific time instance. From here on, $\nabla \cdot \mathbf{B}$ contours and $L^{2}$ norm of $\nabla \cdot \mathbf{B}$ of the underlying scheme refer to the discrete $\nabla \cdot \mathbf{B}$ contours and discrete $L^{2}$ norm of $\nabla \cdot \mathbf{B}$ of the scheme in question. 
Solving the conservative and non-conservative systems comparison: In general, the solution resolution solving the conservative system is almost identical to solving the non-conservative system for the considered test cases, especially on their gas dynamics variables. For Harten-Yee, MUSCL and WENO5, the solution resolution is similar in solving the conservative system as oppose to solving the non-conservative system with the exception of MUSCL (not for all test cases). In some test cases, solving the conservative system, MUSCL exhibit a smaller $L^{2}$-norm $\nabla \cdot \mathbf{B}$ error. Although there is no strong evidence on the advantage of solving the conservative system (aside from not having to evaluate the non-conservative terms) over the non-conservative system for the considered test cases, the possibility of obtaining wrong shock speed/location by the non-conservative system exists for other physical problems [28].

Non-uniform grid and curvilinear grid extension: We note here that the divergence-free property of the base schemes are restricted to uniform Cartesian grids. Although the 3-D curvilinear grid formulation is a straightforward extension of the gas dynamics methodology as in [30], the divergence-free property of the base schemes for non-uniform grid, and general curvilinear grids is not divergence free. If the grid aspect ratio on non-uniform grids are small (i.e., within $10 \%$ of unity), our numerical experiment on a mixing layer computation indicated that divergence free is still possible at for most of the time evolution when the solution is still smooth. After shocks/shears have been formed, complete divergence free is not possible. The resulting $\nabla \cdot \mathbf{B}$ numerical error is, however, many orders of magnitude smaller than the "filter all" option and standard shock-capturing schemes without any divergence cleaning. Thus, the minimization of the $\nabla \cdot \mathbf{B}$ numerical error is also possible by the filter approach if low grid aspect ratio is used in curvilinear grids. An alternative to obtaining a fully divergence free filter scheme for non-uniform curvilinear grids is to employ a staggered grid approach in updating the magnetic field equations for the base scheme step and the corresponding linear filter step. See Sjögreen and Yee [27] for our derivation of the approximate Riemann average states and the eigenvectors in curvilinear grids.

\subsection{MHD Kelvin-Helmholtz Instabilities ( $\gamma=1.4$, Periodic BC)}

The magnetohydrodynamic Kelvin-Helmholtz instabilities have been studied previously by many investigators (see [4] and references cited therein). We have used the set up in [4] which is shown in Fig. 1 [with $\left.\left(u_{x}, u_{y}, u_{z}\right)=(u, v, w)\right]$. At time zero two shear layers are given which are smooth but with strong gradients. A small spatial perturbation is introduced to trigger the instability. The problem is solved to time $T=0.5$. At 


\section{MHD Kelvin-Helmholtz Instability $(\gamma=1.4)$}

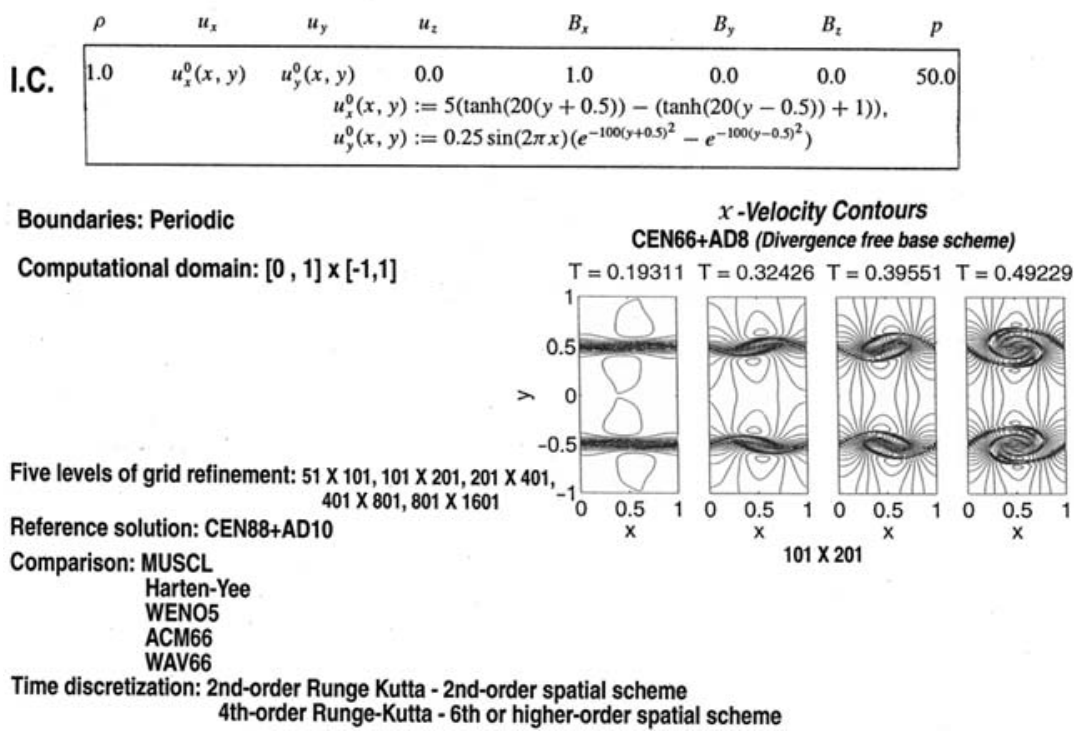

Fig. 1. Problem setup and time evolution of the Kelvin-Helmholtz problem. $x$-velocity contours by CEN66+AD 8 on $101 \times 201$ grid points.

a later time, vortices start to form and gradients become steeper. Snapshots of the time evolution of the $x$-velocity are also shown in Fig. 1 by CEN66+AD8 [sixth-order central with an eighth-order linear numerical dissipation added to the base scheme $(d=0.001)]$. The solution is obtained without the filter step. At stopping time $T=0.5$, the problem is smooth enough that it can be solved by the base scheme alone. Five levels of grid refinement are considered, namely, $51 \times 101,101 \times 201,201 \times 401,401 \times 801$, and $801 \times 1601$.

Figure 2 displays the solution on grids of increasing refinement by the eighth-order central difference with a 10th-order linear dissipation added (CEN88+AD10, $d=0.001)$. Density contours at time $T=0.5$ with 30 equidistant contour levels between 0.4 and 1.2 are used. Again, the CEN88+AD10 solution is obtained by the base scheme CEN88+AD10 alone without the filter step. Computations using $d=0$ (CEN88) are not stable for the entire considered time evolution for all five grids. The CEN88+AD10 solution by solving the non-conservative system is exactly the same as the conservative computation. This is due to the fact that the base scheme CEN88+AD10 alone is divergence-free preserving. The 


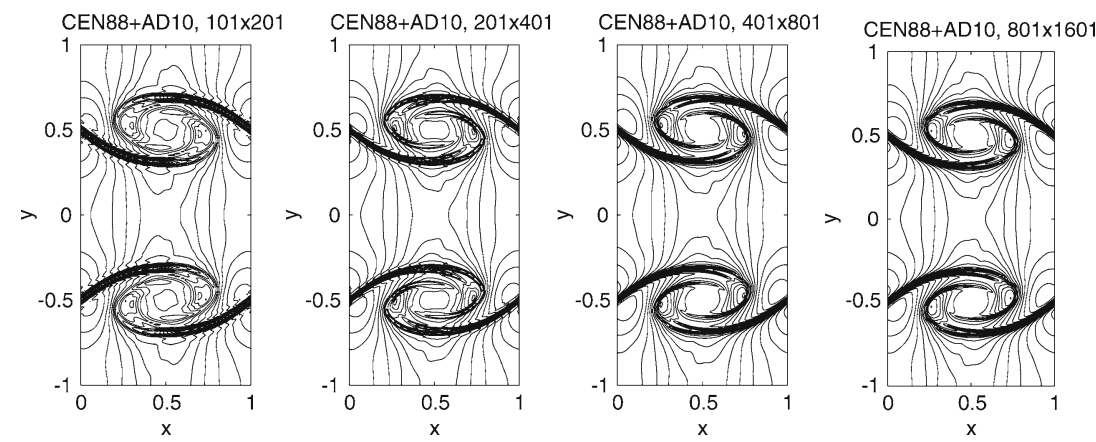

Fig. 2. Grid refinement of CEN88+AD10 solving the conservative system. Density contours at $T=0.5$ for the Kelvin-Helmholtz problem.

sequence of grid refinements shows that the global structure of the flow is in good agreement with the finest grid solution. In order to capture some of the finest structure internal to the vortex, it is necessary to use the $201 \times 401$ grid. The finest grid $801 \times 1601$ by CEN88+AD10 is used as the reference solution.

Although at stopping time $T=0.5$ the problem is smooth enough and there is no need for the more CPU intensive shock-capturing schemes, as the flow evolves at a later time, shock-capturing methods are required. Here, the purpose is to examine the $\nabla \cdot \mathbf{B}$ numerical error when the flow is still smooth using shock-capturing methods. Figure 3 shows the density (left) and $\nabla \cdot \mathbf{B}$ (middle) contours at $T=0.5$, and $L^{2}$-norm of $\nabla \cdot \mathbf{B}$ as a function of time (right) by MUSCL (top row) and WENO5 (bottom row) for three different grids. The same computations by ACM66 and WAV66 using the no filter on $\mathbf{B}$ option are shown in Fig. 4. The $\nabla \cdot \mathbf{B}$ contours with 30 equidistant contour levels between -150 and 150 are used.

Density contours using ACM66, ACM66+AD8 (figures not shown), WAV66, and WAV66+AD8 (figures not shown) exhibit an accuracy similar to CEN88+AD8. There is no gain in solving the conservative over the non-conservative system for these two filter schemes. However, their $\nabla \cdot \mathbf{B}$ numerical errors are very different when using the "no filter on B" option versus the "filter all" option. They are also very different from the standard MUSCL, Harten-Yee and WENO5 schemes. The three standard shock-capturing methods (solving both systems) all exhibit $\nabla \cdot \mathbf{B}$ numerical errors as well as the filter all option of the two filter schemes for the entire time evolution for all five grids. By examining the $\nabla \cdot \mathbf{B}$ contours at $T=0.5$ for all five grids, their $\nabla \cdot \mathbf{B}$ numerical error increases as the grid is refined. For the no filter on $\mathbf{B}$ option, divergence-free preservation is achieved by ACM66 (ACM66+AD8) and WAV66 (WAV66+AD8) for all five grids. 


\section{MHD Kelvin-Helmholtz Instability $(\mathrm{T}=0.5)$ (MUSCL \& WENO5, 201 X 401)}
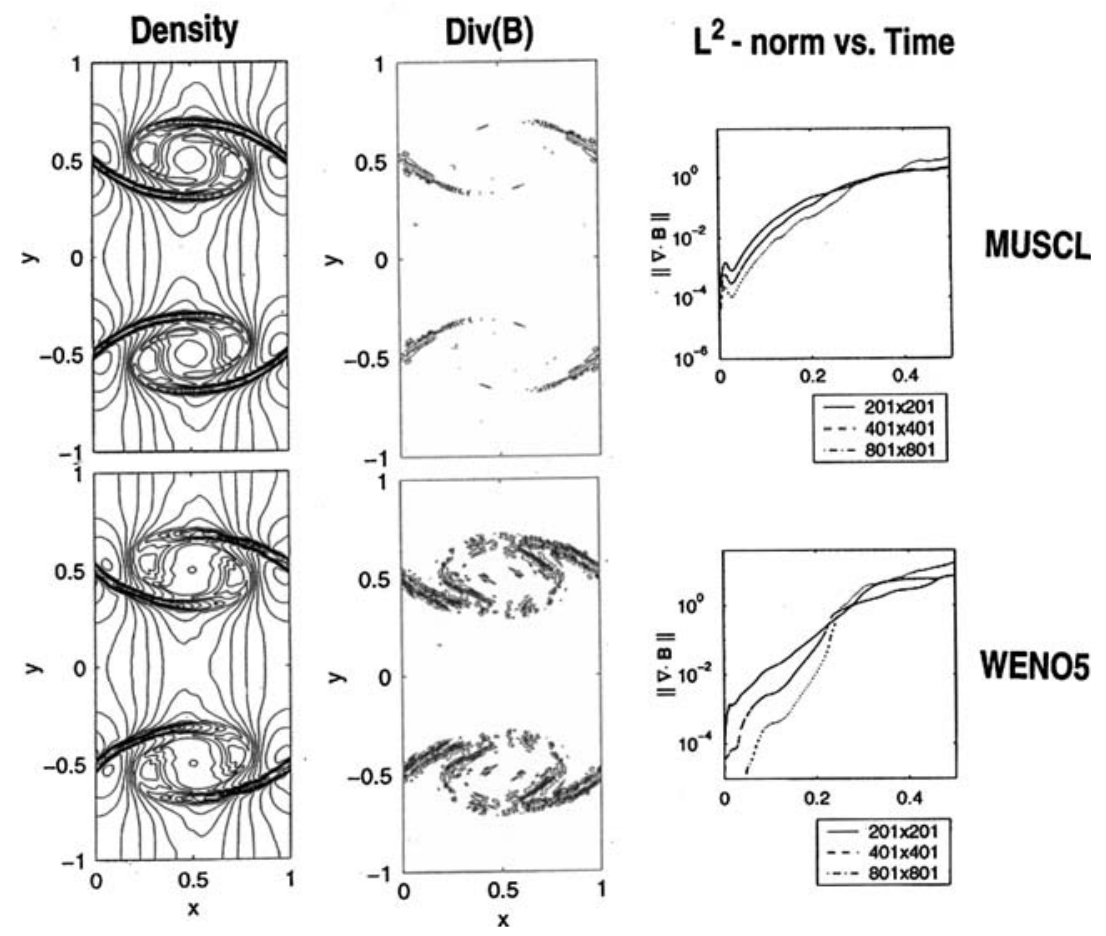

WENO5

Fig. 3. Density (left) and $\nabla \cdot \mathbf{B}$ (middle) contours at $T=0.5\left(201 \times 401\right.$ grid), and $L^{2}$-norm of $\nabla \cdot \mathbf{B}$ as a function of time (right) by MUSCL (top row) and WENO5 (bottom row) for three grids for the Kelvin-Helmholtz problem.

The blank $\nabla \cdot \mathbf{B}$ contours at $T=0.5$ by ACM66 and WAV66 using the no filter on $\mathbf{B}$ option indicate that within the considered contour levels and number of contours, no such contour error was found, whereas MUSCL, Harten-Yee (figure not shown) and WENO5 exhibit the corresponding $\nabla \cdot \mathbf{B}$ numerical errors. The numerical errors at $T=0.5$ are not an isolated case. As a matter of fact, for the entire time evolution, the $L^{2}$-norm $\nabla \cdot \mathbf{B}$ numerical error indicates the superiority of the two filter schemes (no filter on $\mathbf{B}$ ). Note that the $L^{2}$-norm ranges shown are not the same for Figs. 3 and 4.

The CPU time used was considerably larger (around a factor 2.5) for the WENO5 scheme (due to the lower stability limit and higher operations count of WENO5 than the rest of the schemes). MUSCL, Harten-Yee and 


\section{MHD Kelvin-Helmholtz Instability $(T=0.5)$ \\ (Divergence Free ACM66 \& WAV66, 201 x 401)}
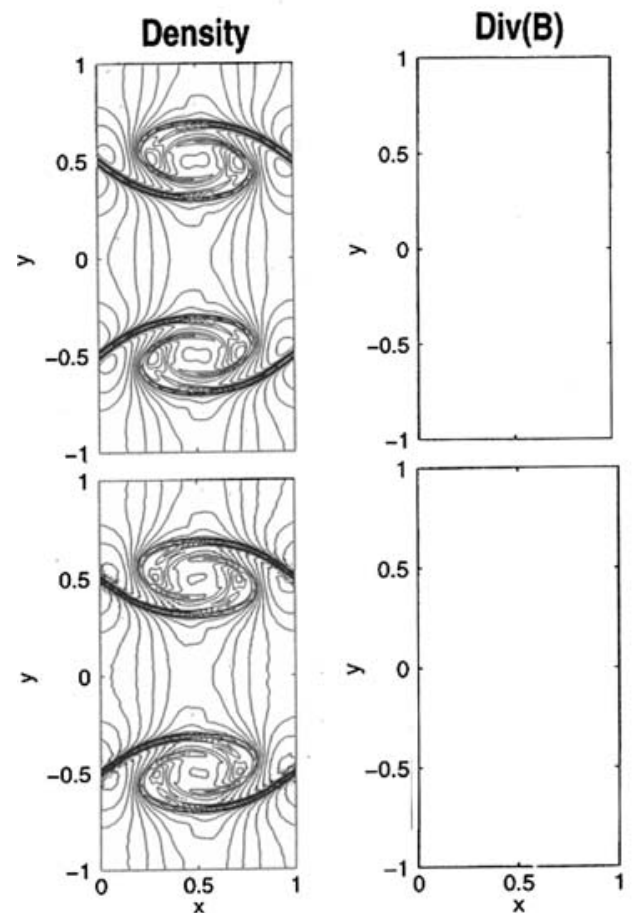

L2 - norm vs. Time
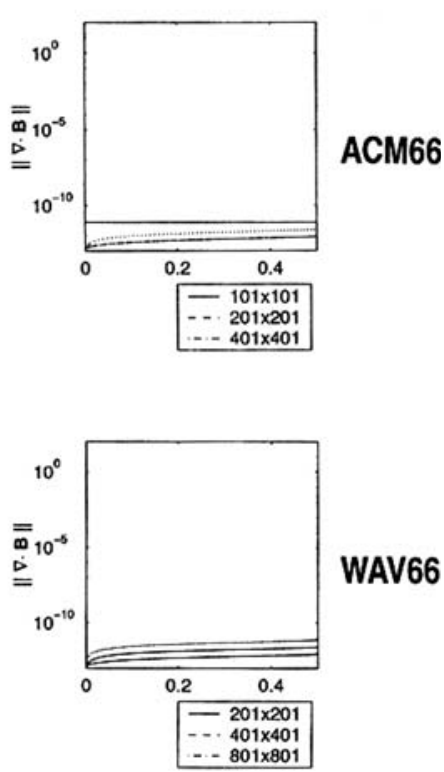

Fig. 4. Density (left) and $\nabla \cdot \mathbf{B}$ (middle) contours at $T=0.5\left(201 \times 401\right.$ grid), and $L^{2}$-norm of $\nabla \cdot \mathbf{B}$ as a function of time (right) by ACM66 (top row) and WAV66 (bottom row) for three grids for the Kelvin-Helmholtz problem.

WENO5 exhibit small oscillations at the outer edges of the vortices as the grid is refined. It is possible to decrease these oscillations by increasing the multi-dimensional entropy fix parameters of the Harten-Yee scheme [31].

\subsection{A 2-D Compressible MHD Riemann Problem $(\gamma=5 / 3)$}

We examine the same 2-D Riemann problem as in [4]. It consists of four constant states at time zero, as shown in Fig. 5. These initial data are chosen so that the magnetic field is divergence free and three of the four 1-D constant states are simple waves as indicated in Fig. 5. The boundaries are treated as open boundaries. The problem is solved on the domain $-1<x<1,-1<y<1$ to time $T=0.2$. Five levels of grid refinement were performed $(101 \times 101,201 \times 201,401 \times 401,801 \times 801$, and $1601 \times 1601)$. 


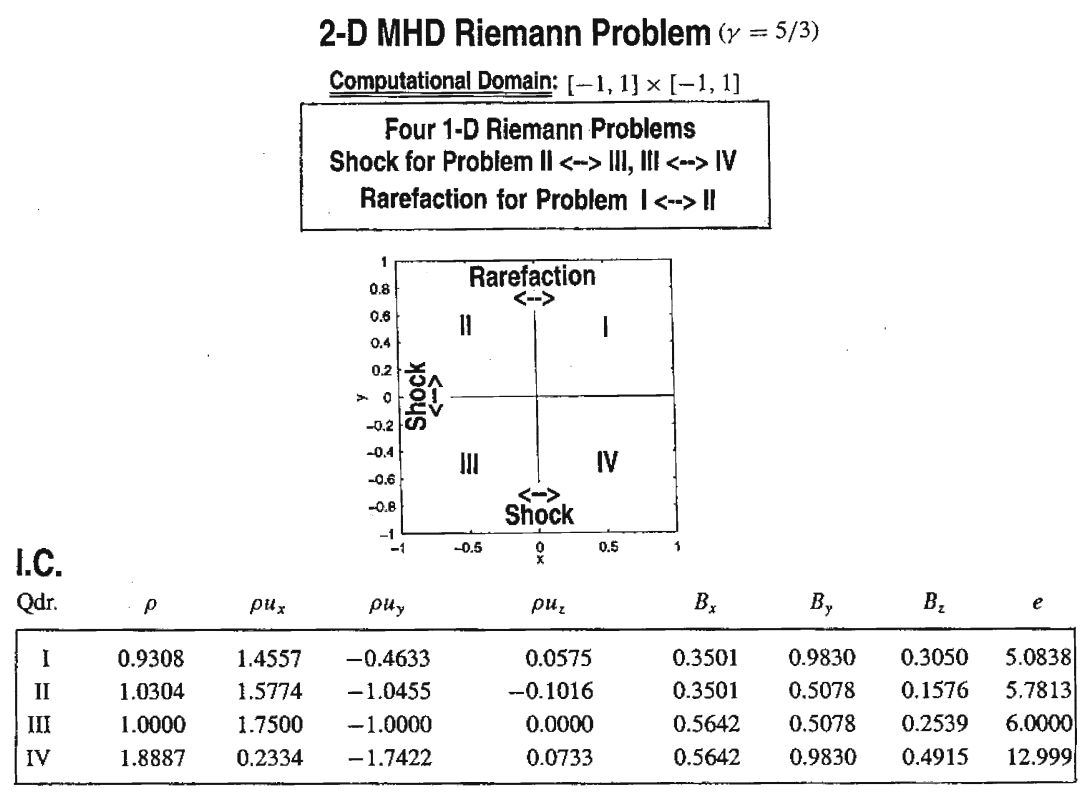

Fig. 5. Schematic of the initial data for the 2-D Riemann problem.

The presence of discontinuities at time zero makes this problem suitable for the study of how $\nabla \cdot \mathbf{B}$ is generated at discontinuities. Grid convergence studies solving conservative (top) and non-conservative (bottom) systems by WENO5 are shown in Fig. 6 for density contours at $T=0.2$ with 40 equally spaced contours between 0.75 and 2.1 . Their density contours are almost identical.

The accuracy in a solution of a Riemann problem away from discontinuities is difficult to improve by increasing the order of the scheme. A large part of the solution is constant, and the structure that develops is affected by low order errors from the discontinuity in the initial data. All five methods can capture shocks within 2-4 grid cells, using the same flux limiter (except WENO5). Their density contours look very similar, even though the $\nabla \cdot \mathbf{B}$ contours or the $L_{2}$-norm of the $\nabla \cdot \mathbf{B}$ numerical errors are all very different.

The effect on $\nabla \cdot \mathbf{B}$ when switching from a non-conservative system to a conservative system is less significant for the Harten-Yee and WENO5 than for MUSCL for the entire time evolution $\left(L^{2}\right.$-norm of $\nabla \cdot \mathbf{B}$ not shown, see [36] for details). $\nabla \cdot \mathbf{B}$ contours for the three methods, MUSCL, Harten-Yee, and WENO5 are displayed in Fig. 7. The $\nabla \cdot \mathbf{B}$ contours use 30 equidistant contour levels between -3.7 and 3.7. Figure 8 shows a 


\section{2-D MHD Riemann Problem}

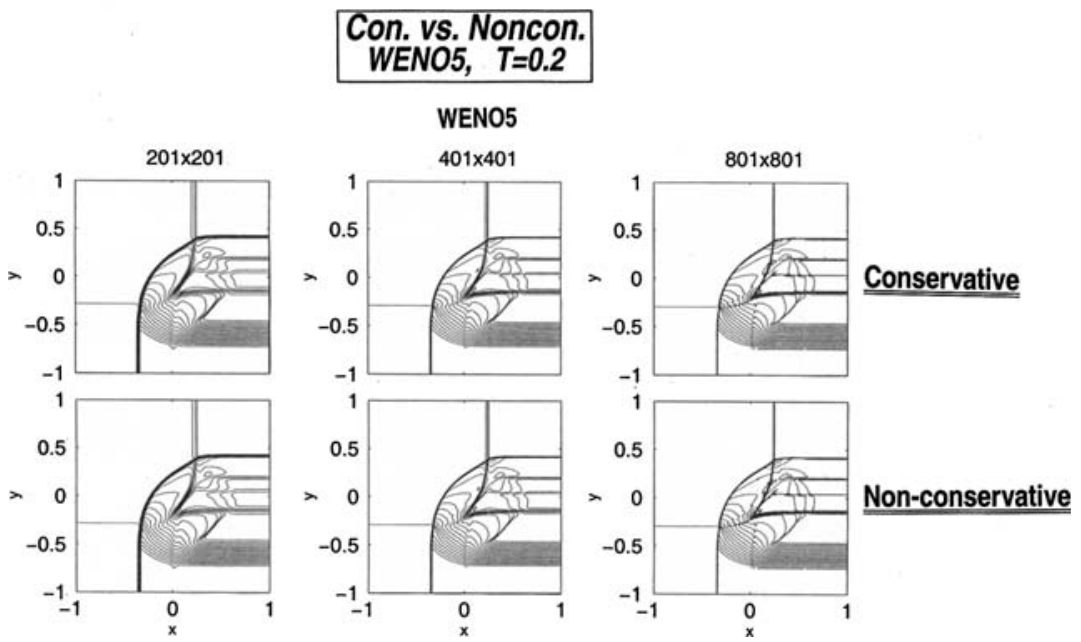

Fig. 6. Grid refinement by WENO5. Density contours solving the conservative (upper row) and non-conservative (lower row) form of the equations for the 2-D Riemann problem.

comparison of the filter all versus no filter on $\mathbf{B}$ options by ACM66+AD8 $(d=0.001)$ for the $\nabla \cdot \mathbf{B}$ contours at $T=0.2$ and the $L^{2}$-norm $\nabla \cdot \mathbf{B}$ time evolution for three grids. Again the blank $\nabla \cdot \mathbf{B}$ contours at $T=0.2$ (bottom right) indicate divergence-free preserving of the no filter on $\mathbf{B}$ option. The $L^{2}$-norm of $\nabla \cdot \mathbf{B}$ at $T=0.2$ confirms the conclusion. As a matter of fact, the four filter schemes (ACM66, ACM66+AD8, WAV66, and WAV66+AD8) all exhibit divergence-free preservation by the no filter on $\mathbf{B}$ option for the entire time evolution. Our study also indicates that the $\nabla \cdot \mathbf{B}$ numerical error at $T=0.2$ increases as the grid is refined by the MUSCL, Harten-Yee, WENO5 and the two filter schemes using the filter all option.

Numerical boundary condition treatments: For problems with non-periodic boundaries, special care has to be taken to avoid generation of $\nabla \cdot \mathbf{B}$ by the numerical boundary condition treatment. This is especially important for spatially higher than second-order base schemes for open boundaries as discussed in the Preliminary Section. When an extrapolation to the outer most boundary point in conjunction with the (SBP) boundary operator obtained from a floating point computation with uncertain round-off properties for the sixth-order base scheme was used, $10^{-6} L^{2}$-norm of $\nabla \cdot \mathbf{B}$ numerical error was generated on the non-periodic boundaries as well. As an alternative, a SBP boundary operator, from [14], was implemented with closed rational expressions for all coefficients. Figure 9 shows the difference in results obtained. The more accurately given SBP operator gave 


\section{2-D MHD Riemann Problem}

MUSCL, Harten-Yee \& WENO5, T=0.2
Con. vs. Noncon.

\section{Div(B) Contours}

Con.
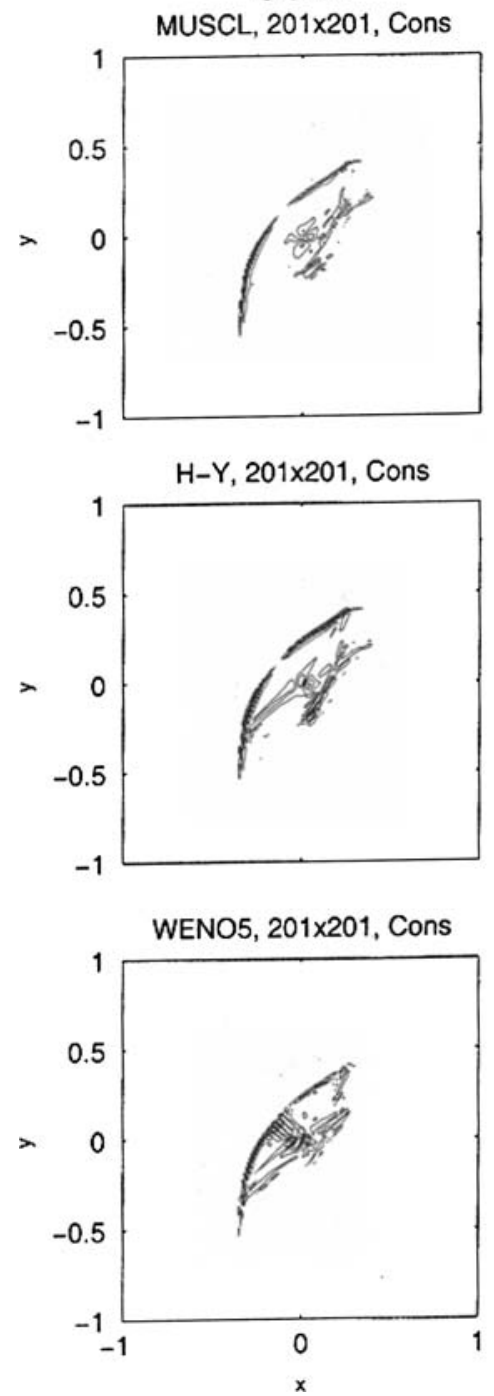

Noncon.

MUSCL, 201×201, Non-cons

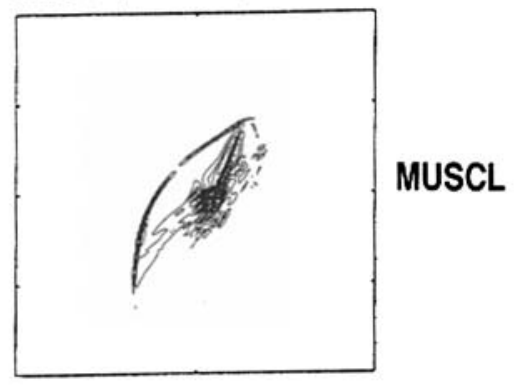

H-Y, 201×201, Non-cons

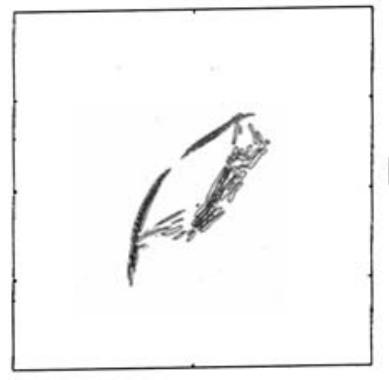

Harten-Yee

WENO5, 201 $\times 201$, Non-cons

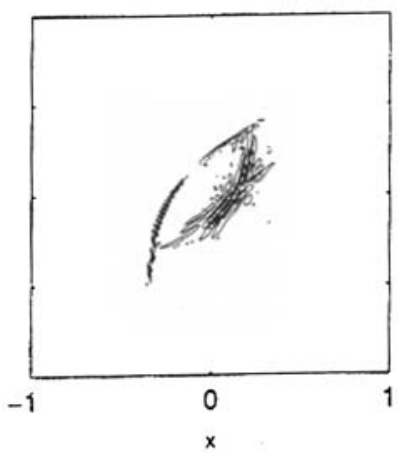

Fig. 7. Comparison of $\nabla \cdot \mathbf{B}$ contours among MUSCL, Harten-Yee and WENO5 using a $201 \times 201$ grid for the 2-D Riemann problem. 


\section{2-D MHD Riemann Problem \\ (Filter All) vs. (No Filter on B) \\ $A C M 66+A D 8$}

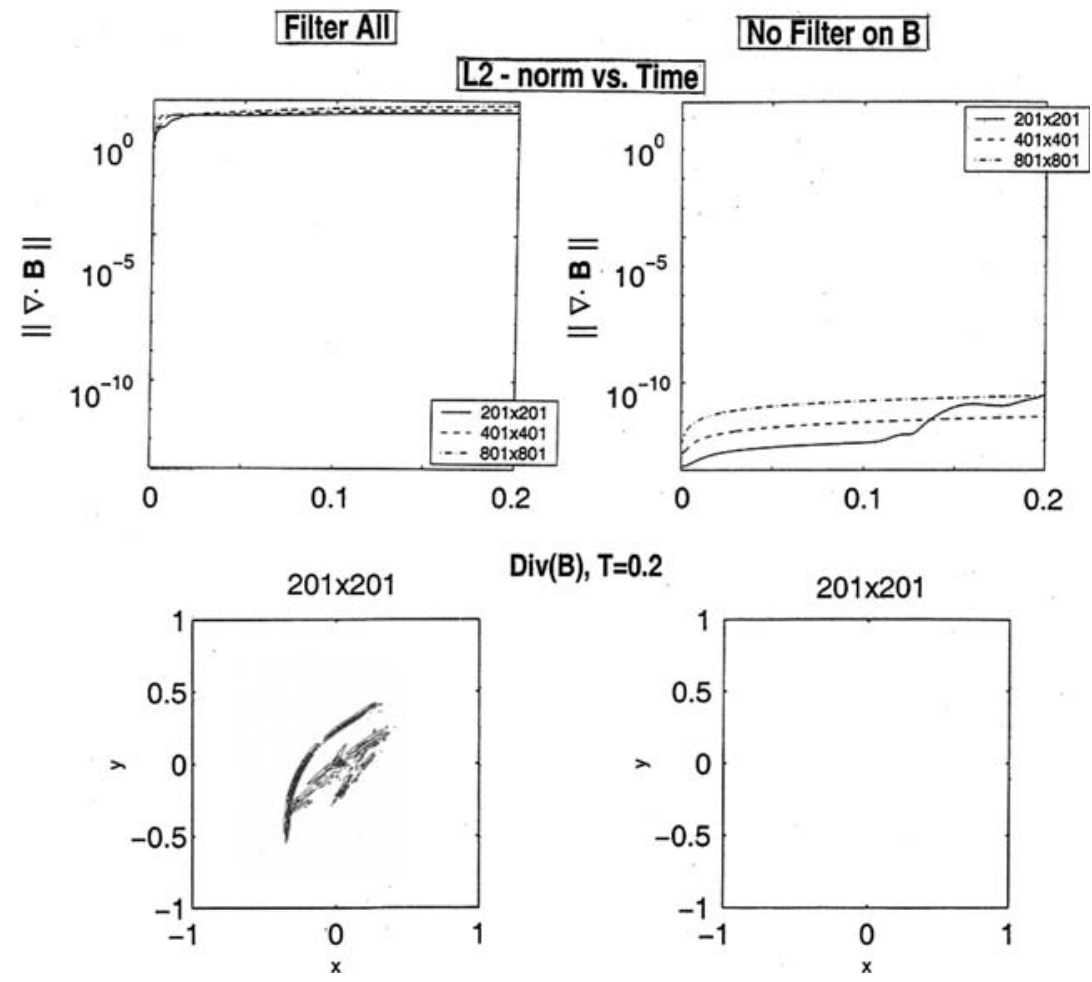

Fig. 8. $L^{2}$-norm of $\nabla \cdot \mathbf{B}$ versus. time and $\nabla \cdot \mathbf{B}$ contours at $T=0.2$ by ACM66+AD8, $d=0.001$, no filter on $\mathbf{B}$ (left) and filter all (right) using $201 \times 201,401 \times 401$, and $801 \times 801$ grid points for the 2-D Riemann problem.

a considerably smaller $\nabla \cdot \mathbf{B}$ generation on the boundary. It is noted that the grid stencil of the SBP boundary operator in [14] is smaller than the SBP boundary operator in $[15,16]$. Since the flow near both the $x$ and $y$ directions boundaries are either smooth or uniform (aside from a few isolated discontinuities), the SBP boundary operator has very small effect on the generation of $\nabla \cdot \mathbf{B}$ numerical error through the boundaries. The fourth test problem consists of complex wave interactions on both the $x$ and $y$ boundary directions at later time evolution, using a wide grid stencil SBP boundary operator will exhibit larger $\nabla \cdot \mathbf{B}$ numerical error as can be seen later. 


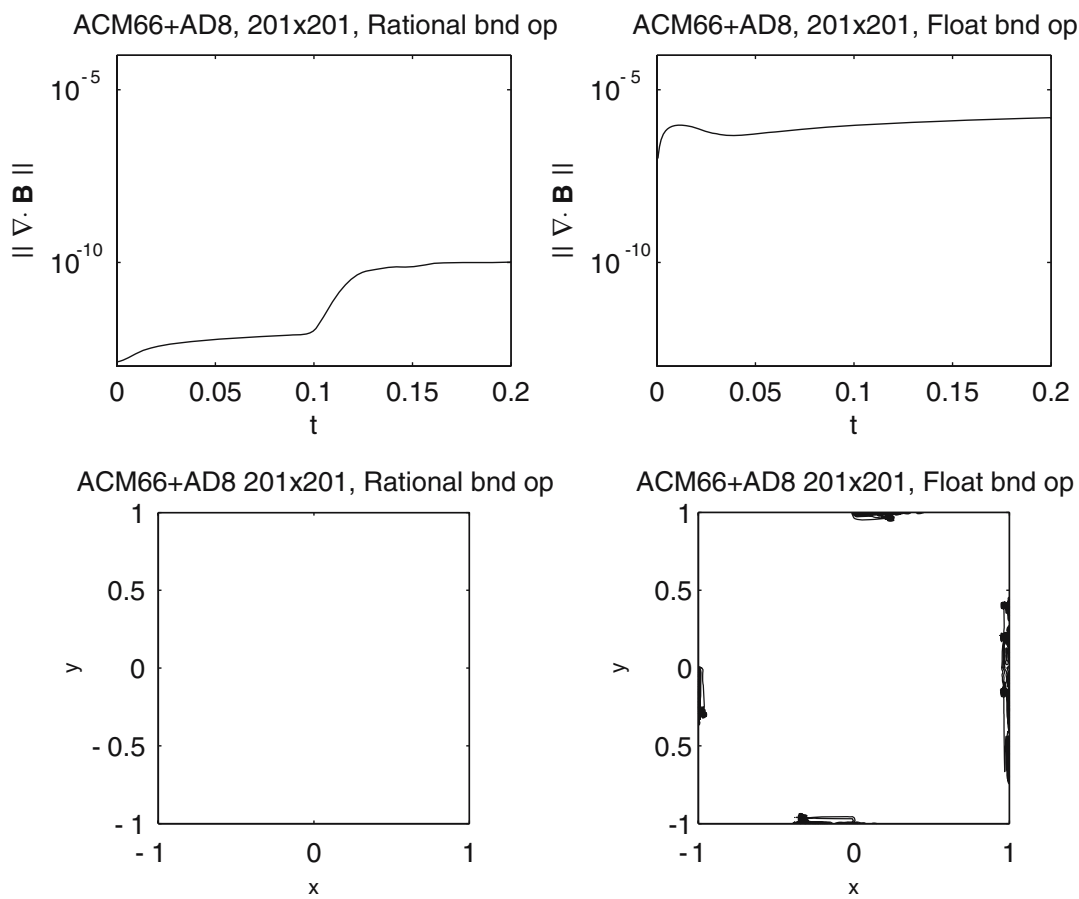

Fig. 9. ACM66 $+\mathrm{AD} 8(d=0.001$, no filter on $\mathbf{B}$ option) computations for the 2-D Riemann problem. $L^{2}$-norm of $\nabla \cdot \mathbf{B}$ versus time, and $\nabla \cdot \mathbf{B}$ contours at $T=0.2$. SBP operator given as rational numbers (left) and SBP operator computed by a lengthy floating point computation (right) using a $201 \times 201$ grid.

\subsection{Compressible Orszag-Tang Vortex $(\gamma=5 / 3$, Periodic BC)}

The 2-D compressible Orszag-Tang vortex problem $[5,6]$ consists of periodic boundary conditions with smooth initial data as shown in Fig. 10. The computational domain is $0<x<2 \pi, 0<y<2 \pi$. This popular test case is a compressible MHD version of the original incompressible MHD Orszag-Tang vortex problem [18].

The computation stops at time $T=3.14(\approx \pi)$, when discontinuities and complicated flow interactions have formed. Density contours with 30 equally spaced contours between 0.9 and 6.1 , and $\nabla \cdot \mathbf{B}$ contours with 30 equally spaced contours between -30 and 30 are used for illustration. Again, the same five levels of grid refinement study as the 2-D Riemann problem were performed on all five methods. Density contours by WAV66+AD8 at $T=3.14$ using "Filter all" and "no filter on B" are 


\section{Compressible Orszag-Tang Vortex $(\gamma=5 / 3)$}

I.C.

$\left(\begin{array}{c}\rho \\ u \\ v \\ w \\ p \\ B_{x} \\ B_{y} \\ B_{z}\end{array}\right)=\left(\begin{array}{c}25 / 9 \\ -\sin y \\ \sin x \\ 0 \\ 5 / 3 \\ -\sin y \\ \sin 2 x \\ 0\end{array}\right)$

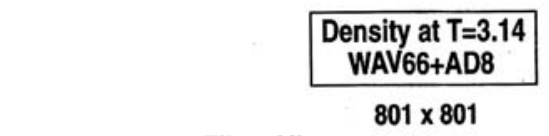

BC: Periodic

Domain: $0<x<2 \pi$

$0<y<2 \pi$

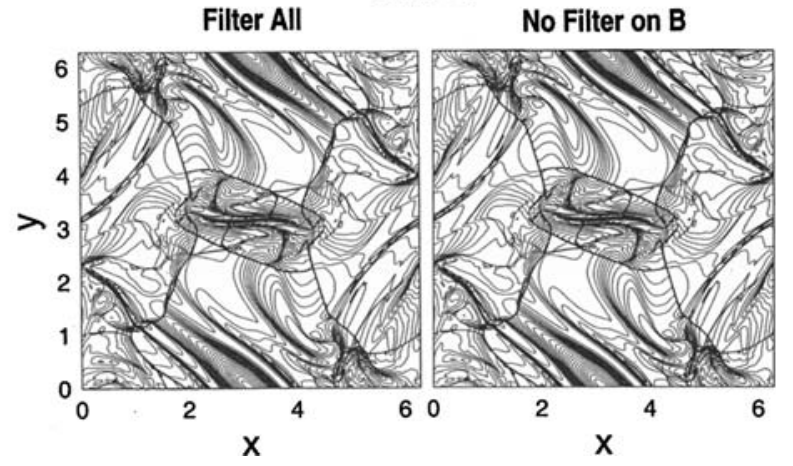

Fig. 10. Schematic, problem setup and density contours by WAV66+AD8 for the OrszagTang problem using a $801 \times 801$ grid at time $T=3.14$.

also shown in Fig. 10. The density contours are almost identical. Their $\nabla \cdot \mathbf{B}$ numerical error are very different as we will discussed next.

Figures $11-15$ show the comparison of $\nabla \cdot \mathbf{B}$ contours at $T=3.14$ and $L^{2}$-norm of $\nabla \cdot \mathbf{B}$ among the five methods. Figures $11-13$ also show the comparison among MUSCL, Harten-Yee and WENO5 solving both systems using a fine grid of $801 \times 801$. In addition, Figs. 14 and 15 compare the "no filter on B" option with the "Filter all" option. Note that the $L^{2}$-norm range for Figs. $11-13$ is different from Figs. 14 and 15. Care must be taken to interpret their corresponding $L^{2}$-norm $\nabla \cdot \mathbf{B}$ errors. Divergence-free preservation is achieved by WAV66+AD8 $(d=0.001)$ and ACM66+AD8 $(d=0.001)$ using the no filter on B option.

The MUSCL scheme applied to the non-conservative equations produces a considerably larger error in $\nabla \cdot \mathbf{B}$ than does the same method applied to the conservative equations. The $\nabla \cdot \mathbf{B}$ development for HartenYee and WENO5 shows less difference between the conservative and the non-conservative equations. The size of the generated $\nabla \cdot \mathbf{B}$ is similar for both equations, and somewhat more spread out for the non-conservative equations.

The $\nabla \cdot \mathbf{B}$ contour error at $T=3.14$ by ACM66+AD8 with the filter all option is larger than WAV66+AD8, Harten-Yee and WENO5, but smaller than MUSCL. The behavior of WAV66 $(d=0)$ and ACM66 $(d=0)$ is 

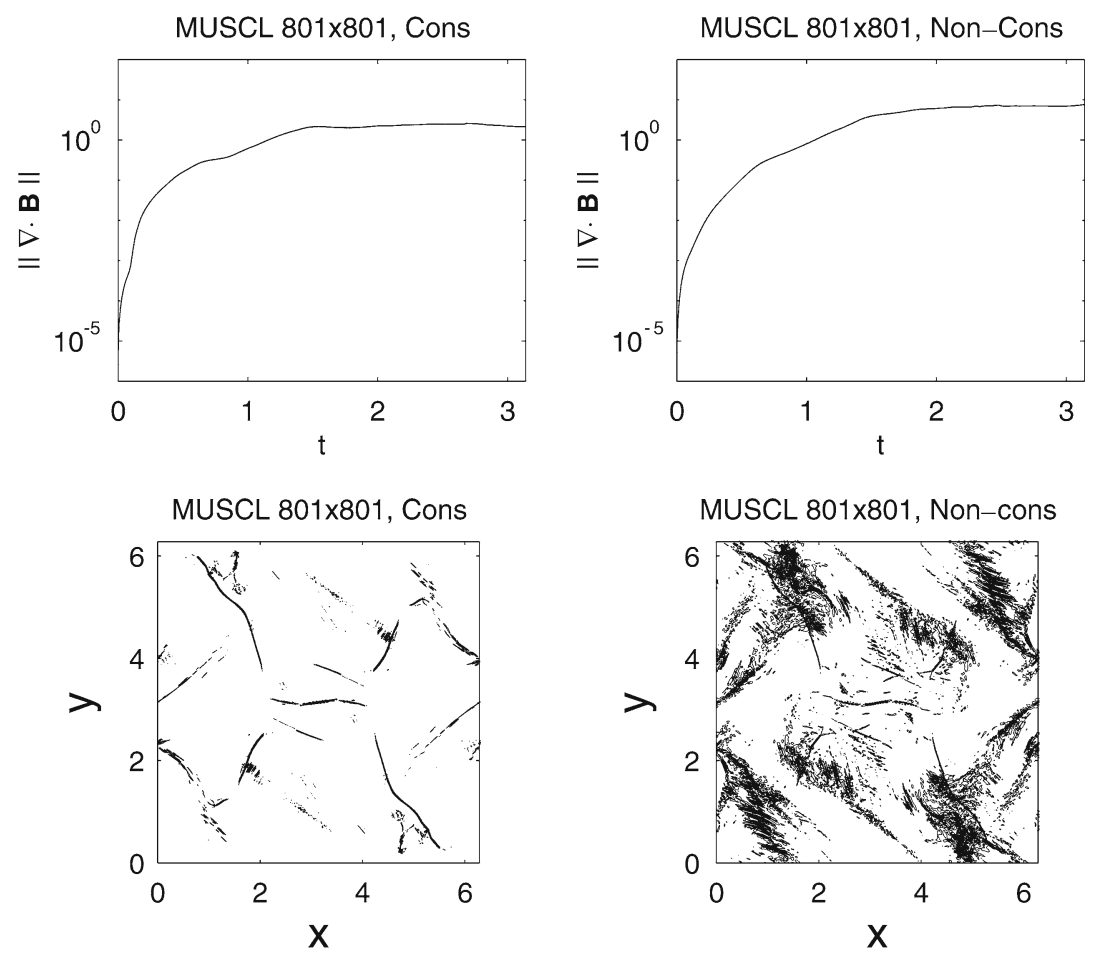

Fig. 11. $L^{2}$-norm of $\nabla \cdot \mathbf{B}$ in time (top row) and $\nabla \cdot \mathbf{B}$ contours at $T=3.14$ (bottom row ) by MUSCL for the Orszag-Tang problem, conservative (left) and the non-conservative (right) equations using a $801 \times 801$ grid.

similar to WAV66+AD8 and ACM+AD8, respectively. It is interesting to point out that divergence free is also possible for the "Filter all" option by WAV66+AD8 for $T<0.7$, whereas the ACM66+AD8 loses its divergencefree preservation at a much earlier time. This is due to the fact that the WAV-filter scheme sensor turns off the non-linear filter at regions of very smooth flow (in this case for $T<0.7$ ) whereas the ACM-filter only reduces the strength of the non-linear filter for $T<0.7$ for the filter all option. For this problem, WENO5 exhibits a lower $L^{2}$-norm error for $T<0.6$ in solving the conservative system over the non-conservative system. Divergence free is also possible by WENO5 at very early stages of the time evolution for solving both systems.

The resolution of the global structure of the density contours is well captured by all five methods. However, small fine structures were captured by the ACM-filter and WAV-filter schemes on a $101 \times 101$ grid, and not 


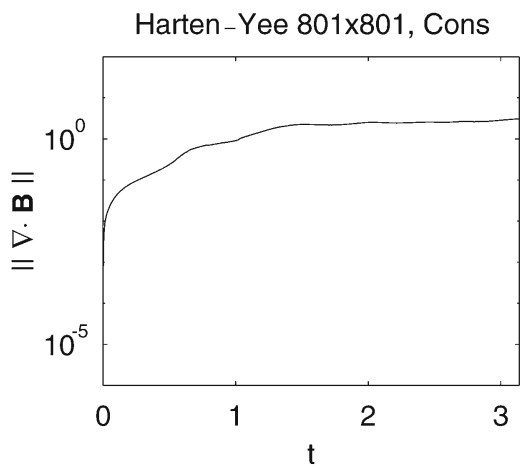

Harten-Yee 801x801, Cons

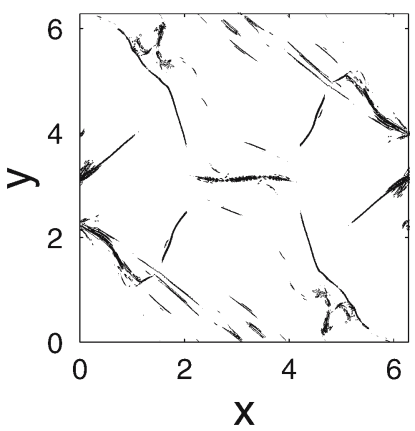

Harten-Yee $801 \times 801$, Non-Cons

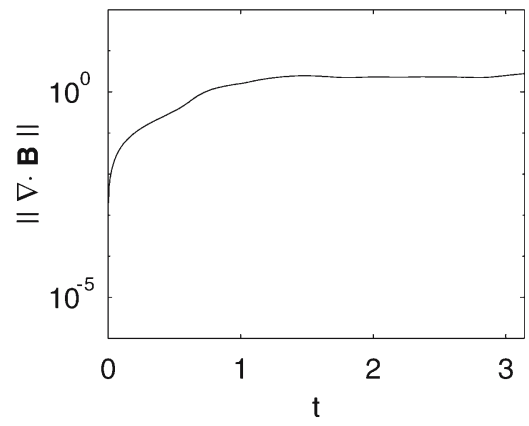

Harten-Yee 801x801, Non-cons

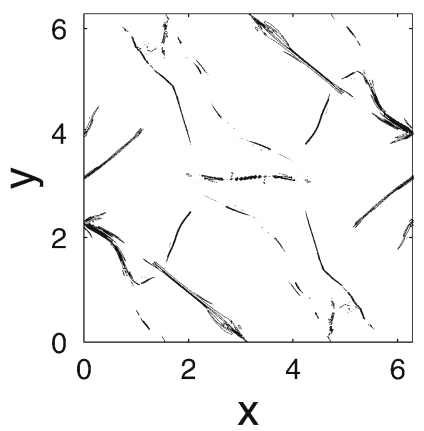

Fig. 12. $L^{2}$-norm of $\nabla \cdot \mathbf{B}$ in time (top row) and $\nabla \cdot \mathbf{B}$ contours at $T=3.14$ (bottom row) by Harten-Yee for the Orszag-Tang problem, conservative (left) and the non-conservative (right) systems using a $801 \times 801$ grid.

by MUSCL, Harten-Yee and WENO5 using the same grid. Similar to the first two test cases, the $\nabla \cdot \mathbf{B}$ numerical error at $T=3.14$ increases as the grid is refined by MUSCL, Harten-Yee, WENO5 and the two filter schemes using the filter all option.

\subsection{A Planar Shock Interacting with a Magnetic Cloud $(\gamma=5 / 3$, Supersonic Inflow and Open Boundaries)}

The fourth test problem is a planar shock interacting with a magnetic cloud studied in $[5,6]$. This is a more challenging problem to simulate due to the stiffness of the flow with rapidly developed complex wave interactions close to the boundaries. The same initial configuration as in [28] is considered here. The computational domain is the square $0<x<1,0<$ $y<1$. A planar shock is initially situated at $x=0.6$ and move towards the right. In a circular region with center at $(0.8,0.5)$ and radius 0.15 , a 

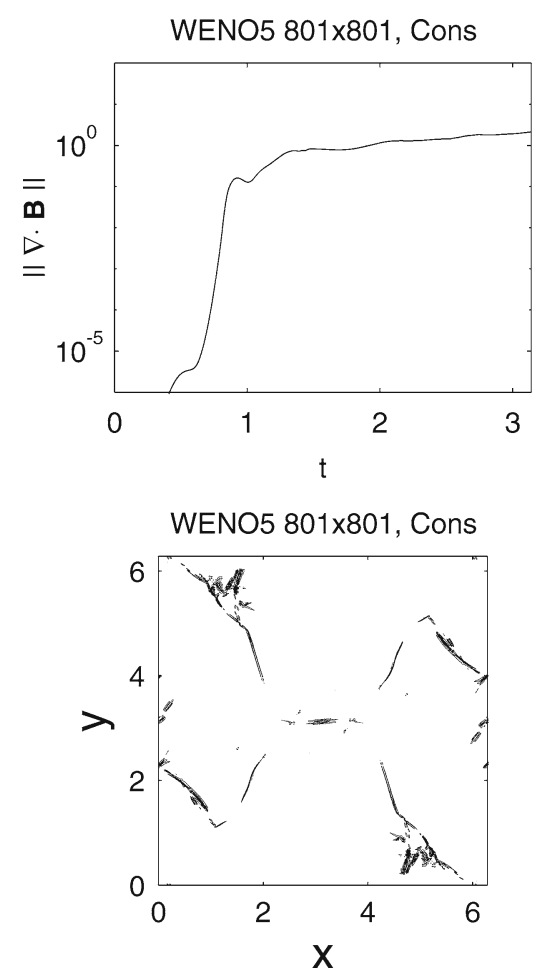

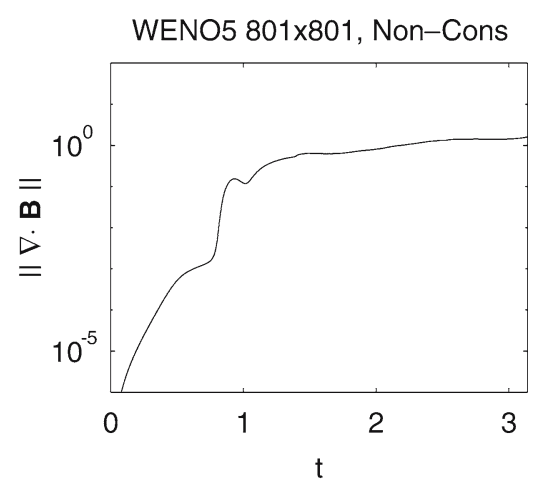

WENO5 801x801, Non-cons

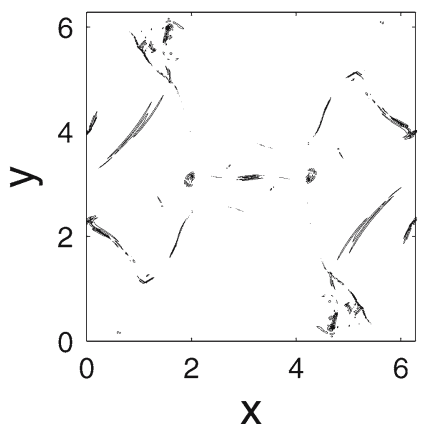

Fig. 13. $L^{2}$-norm of $\nabla \cdot \mathbf{B}$ in time (top) and $\nabla \cdot \mathbf{B}$ contours at $T=3.14$ (bottom) by WENO5 for the Orszag-Tang problem solving the conservative (left) and the non-conservative (right) systems using a $801 \times 801$ grid.

state of increased density (i.e., the cloud) is given. The problem setup and schematic of the initial condition are shown in Fig. 16. The flow velocity is directed in the negative $x$-direction, and the cloud will move to the left as indicated on Fig. 16. The right boundary is supersonic inflow, where the right state is imposed. The other boundaries are open boundaries. The same five levels of grid refinement for the last two test cases were performed on this shock/cloud interaction problem. Density contours with 50 equidistant contours in $\log$ scale from $\log (0.99)$ to $\log (48)$, and the $\nabla \cdot \mathbf{B}$ contours with 30 equidistant contours between -500 and 500 are used. Figure 16 also shows the density contours by WENO5 at $T=0.06$ using a $801 \times 801$ grid.

Figures 17 and 18 show the comparison between WENO5 (solving both systems $)$ and WAV66+AD8 $(d=0.001)$ in terms of $L^{2}$-norm of $\nabla \cdot \mathbf{B}$ for three grids and the $\nabla \cdot \mathbf{B}$ contours at $T=0.06$. In addition 
WAV66+AD8 801x801, no filter on B WAV66+AD8 801x801, filter on all components
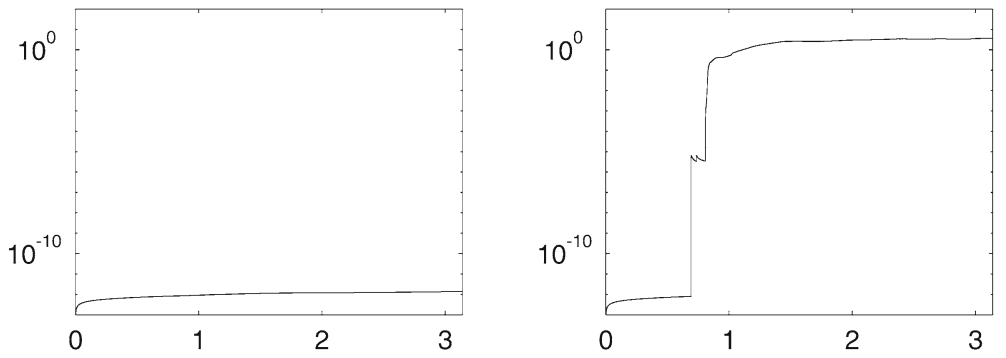

WAV66+AD8 801 $\times 801$, no filter on B

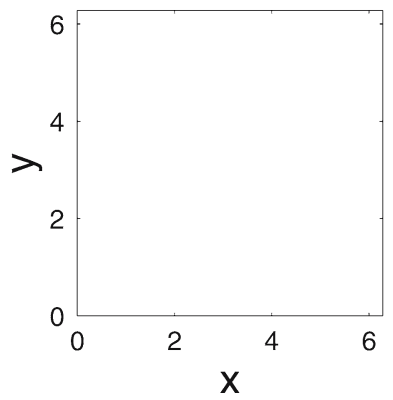

WAV66+AD8 801x801, filter on all components

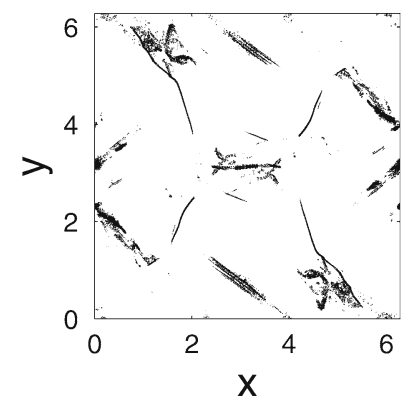

Fig. 14. WAV66+AD $8 L^{2}$-norm of $\nabla \cdot \mathbf{B}$ in time (top row), $\nabla \cdot \mathbf{B}$ contours at $T=3.14$ (bottom row) by WAV66+AD8 for the Orszag-Tang problem. No non-linear filter on B (left) and non-linear filter on all components (right) using a $801 \times 801$ grid.

Fig. 18 shows the comparison between filter all and no filter on $\mathbf{B}$ by WAV66+AD8. Figure 19 shows similar computations by ACM66+AD8 $(d=0.001)$ using a $801 \times 801$ grid. Figures 20 and 21 show the $L^{2}$ norm of $\nabla \cdot \mathbf{B}$ comparison among MUSCL, WENO5, ACM66+AD8 and WAV66+AD8. Note that the range of the $L^{2}$-norm in Figs. 17 and 20 is different from Figs. 18, 19, and 21.

For this test case, the $L^{2}$-norm error by the three standard shockcapturing methods and the two filter schemes using the filter all options are similar. In most parts of the time evolution, above unity error was obtained. Their $\nabla \cdot \mathbf{B}$ contour errors at $T=0.06$ are different (different distributions of $\nabla \cdot \mathbf{B}$ ). In addition, their $\nabla \cdot \mathbf{B}$ numerical errors at $T=0.06$ increase as the grid is refined. The filter all options by the filter schemes exhibit a larger $\nabla \cdot \mathbf{B}$ contour error at $T=0.06$ than Harten-Yee and WENO5.

The different distribution of $\nabla \cdot \mathbf{B}$ can be seen by examining the respective figures. For example, WENO5 is better at suppressing $\nabla \cdot \mathbf{B}$ 


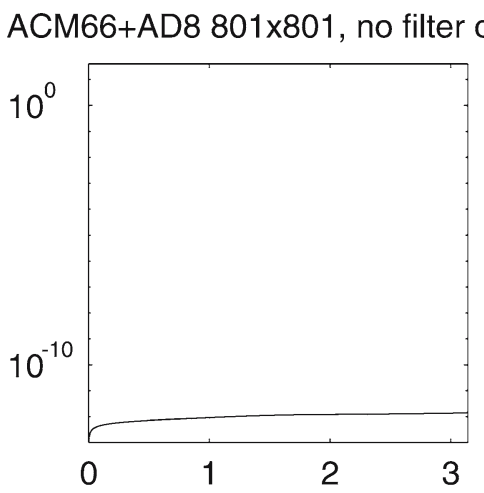

\section{B}

ACM66+AD8 801x801, filter all

ACM66+AD8 801x801, no filter on B
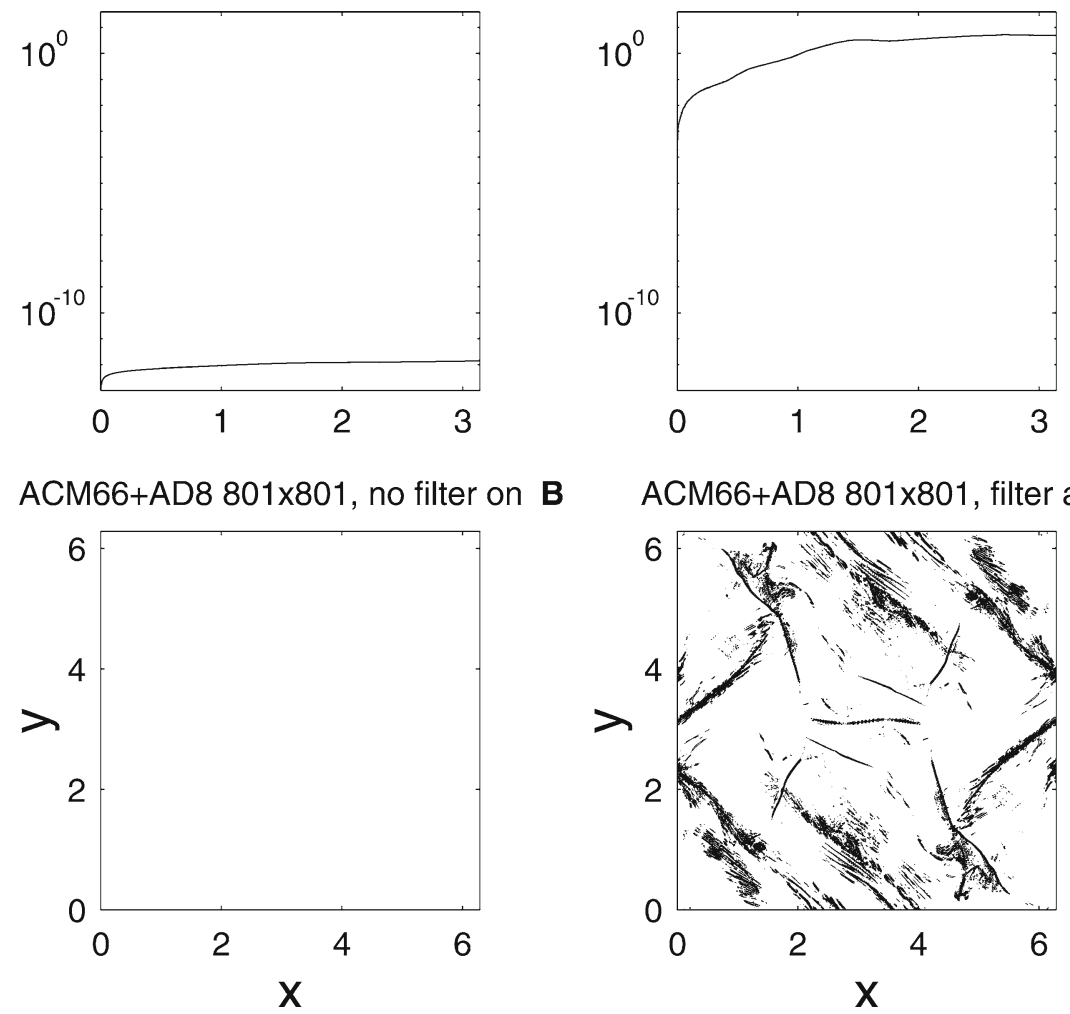

ACM66+AD8 801x801, filter all

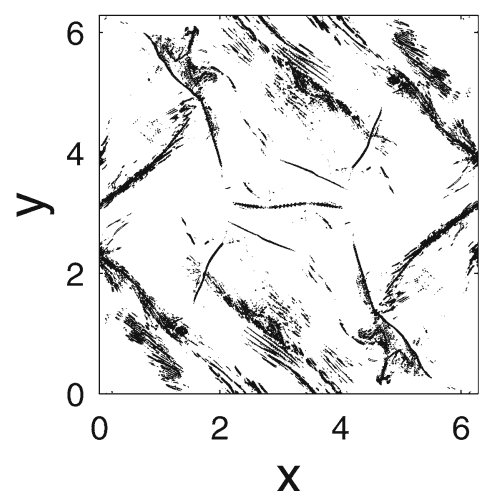

Fig. 15. $L^{2}$-norm of $\nabla \cdot \mathbf{B}$ in time (top row) and $\nabla \cdot \mathbf{B}$ contours at $T=3.14$ (bottom row) by ACM66+AD8 for the Orszag-Tang problem. No non-linear filter on B (left) and non-linear filter on all components (right) using a $801 \times 801$ grid.

generation at the main shock. The non-conservative equations evolve $\nabla \cdot \mathbf{B}$ with the streamlines, which for this problem are directed towards the negative $x$-direction. Therefore, a larger $\nabla \cdot \mathbf{B}$ numerical error is seen to the bottom left of Fig. 17. For the MUSCL and Harten-Yee schemes (figures not shown), solving the conservative system is slightly better than solving the non-conservative system by examining their $\nabla \cdot \mathbf{B}$ contours at $T=0.06$.

For both the WAV66+AD8 and ACM66+AD8 using the no filter on $\mathbf{B}$, perfect $\nabla \cdot \mathbf{B}$ preservation is only obtained up to a certain time $(T=$ 0.04). The increase in the norm of $\nabla \cdot \mathbf{B}$ is caused by boundary effects. A SBP difference boundary operator is used. Due to the wide grid stencil of the SBP boundary difference operator in conjunction with the need to use an extrapolation to the outermost open boundary point, $\nabla \cdot \mathbf{B}$ is not 


\section{Supersonic Shock Interacting with a Magnetic Cloud $(\gamma=5 / 3)$}

I.C.

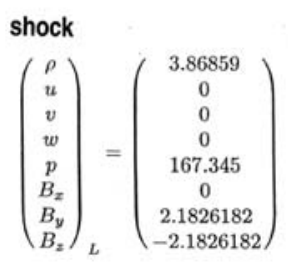

cloud

$$
\left(\begin{array}{c}
\rho \\
u \\
v \\
w \\
p \\
B_{x} \\
B_{y} \\
B_{z}
\end{array}\right)=\left(\begin{array}{c}
10 \\
-11.2536 \\
0 \\
0 \\
1 \\
0 \\
0.56418958 \\
-0.56418958
\end{array}\right)
$$

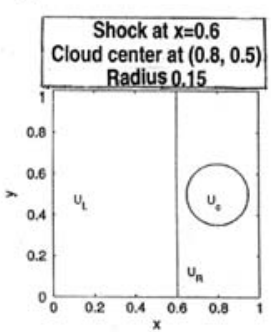

Domain: $[0,1] \times[0,1]$

Density at $\mathrm{T}=0.06$ by WENO5, $801 \times 801$, Cons.

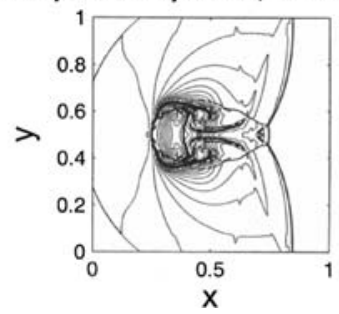

Fig. 16. Problem setup and schematic of the initial data of the MHD shock/cloud interaction problem. Density contours by WENO5 solving the conservative system using a $801 \times$ 801 grid.

preserved. The effect is only seen when the solution is non-trivial on the boundary where complex wave interactions are taking place in both directions of the open boundaries.

Although divergence-free preservation by ACM66+AD8 and WAV66+ AD8 is not possible for $T>0.04$ by the "no filter on B" option, the $L^{2}$ norm for this option is at least an order of magnitude smaller than the "Filter all" option and the three standard shock-capturing schemes when $T>0.04$. Figures 20 and 21 show the distinct lower $L^{2}$-norm error levels than the MUSCL and WENO5. Note again that the range of the $L^{2}$-norm in Figs. 17 and 20 is different from Figs. 18, 19, and 21. Care is needed when comparing the results.

The solution by ACM66+AD8 (figure not shown) solving either the conservative or non-conservative system is slightly different from the other four methods. A very weak discontinuity is seen to originate from the cloud which hits the lower boundary at around $x=0.6$. This discontinuity is not present in any of the other computed solutions. Furthermore, the main shock is in a slightly different position than in the rest of the methods. The solution behavior of ACM66+AD8 is similar for both the filter 


\section{Supersonic Shock Interacting with a Magnetic Cloud WENO5, T=0.06 \\ Con. vs. Noncon}

Con.

Noncon.
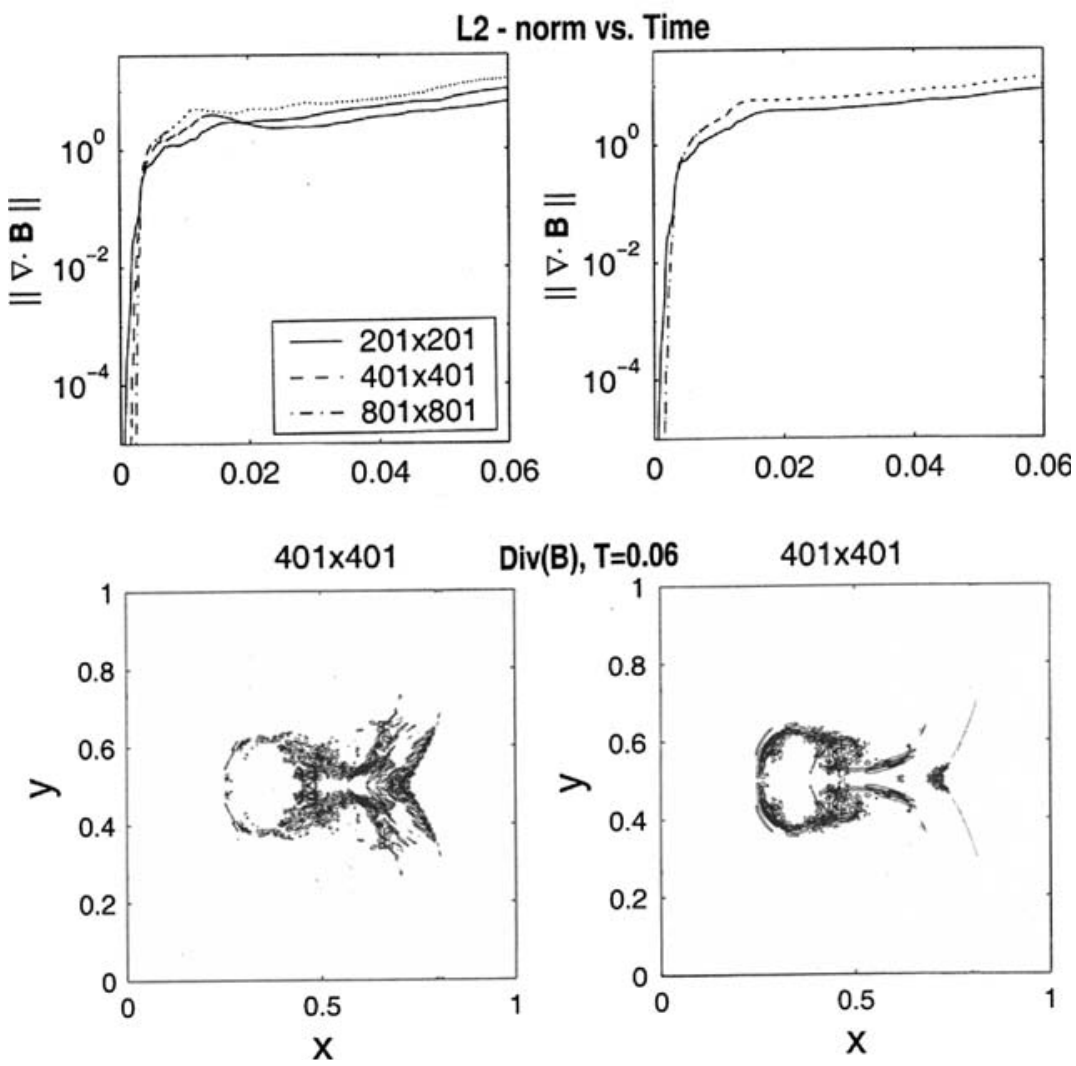

$\operatorname{Div}(B), T=0.06 \quad 401 \times 401$

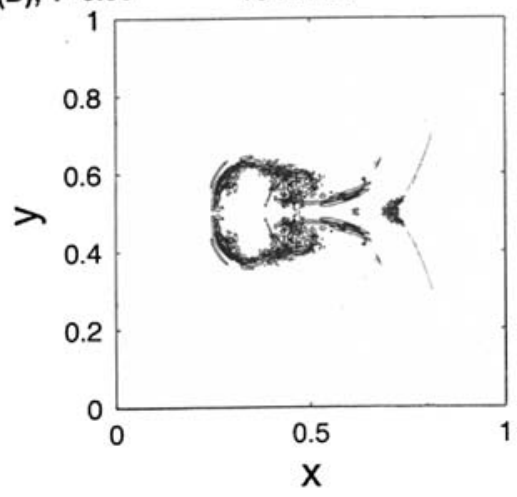

Fig. 17. Grid convergence study of $L^{2}$-norm of $\nabla \cdot \mathbf{B}$, and $\nabla \cdot \mathbf{B}$ contours at $T=0.06$ by WENO5 using a $401 \times 401$ grid, conservative (left) and non-conservative (right) for the shock/cloud interaction problem.

all and no filter on $\mathbf{B}$ options. The reason for this discrepancy is under investigation. The wide grid stencil of the SBP boundary operator might play a role.

Since this is a very stiff problem, very small CFL is required. For the finer grid, in order to obtain a stable solution by WENO5 ( $C F L=0.1$, the CPU time is more than an order of magnitude greater than for the 


\section{Supersonic Shock Interacting with a Magnetic Cloud (Filter All) vs. (No Filter on B) WAV66+AD8}

(Filter All)

(No Filter on B)

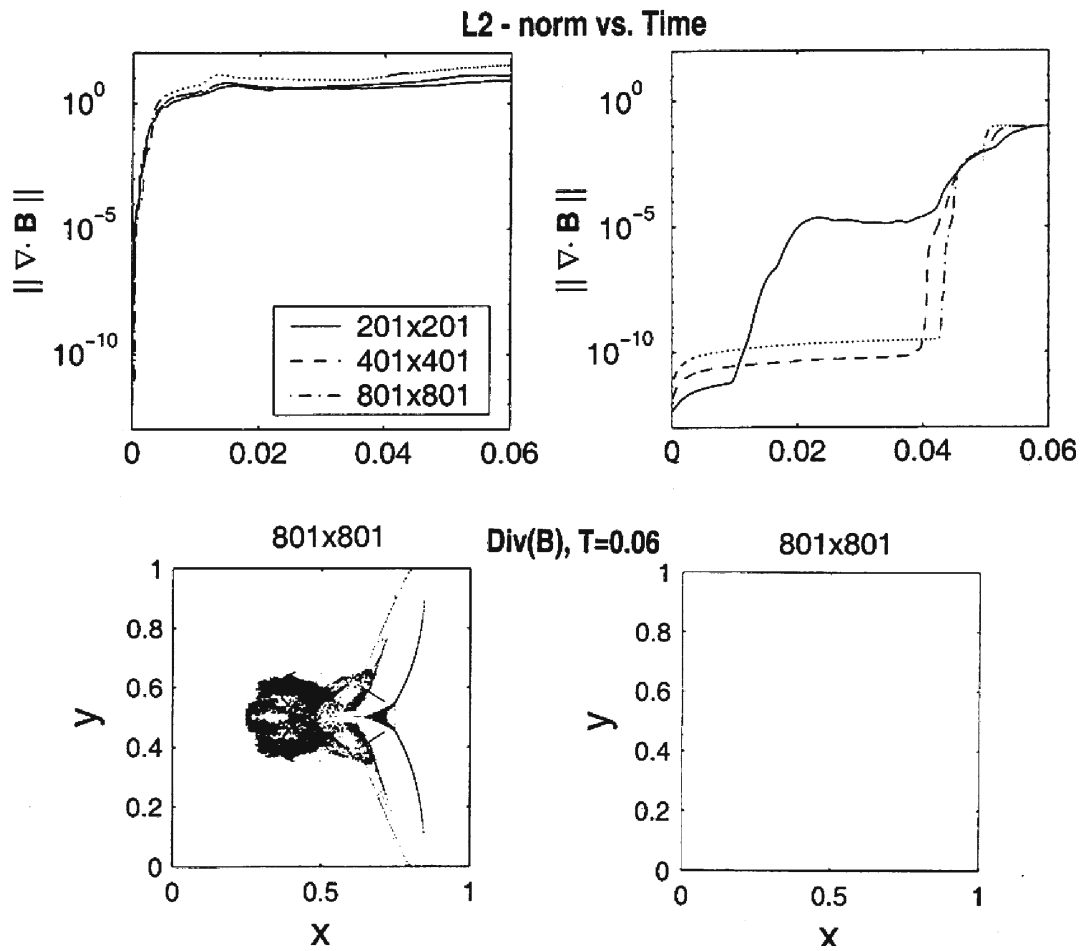

Fig. 18. Grid convergence study of $L^{2}$-norm of $\nabla \cdot \mathbf{B}$, and $\nabla \cdot \mathbf{B}$ contours at $T=0.06$ by WAV66+AD8 using a $801 \times 801$ grid, filter all (left) and no filter on $\mathbf{B}$ (right) for the shock/cloud interaction problem.

Harten-Yee and MUSCL schemes, and many times more CPU time than the ACM and WAV-filter schemes. This is partially due to a lower stability limit of WENO5 than the rest of the schemes.

\section{CONCLUDING REMARKS}

A natural and efficient high order finite difference filter approach in the sense of not needing traditional divergence cleaning for the minimization 

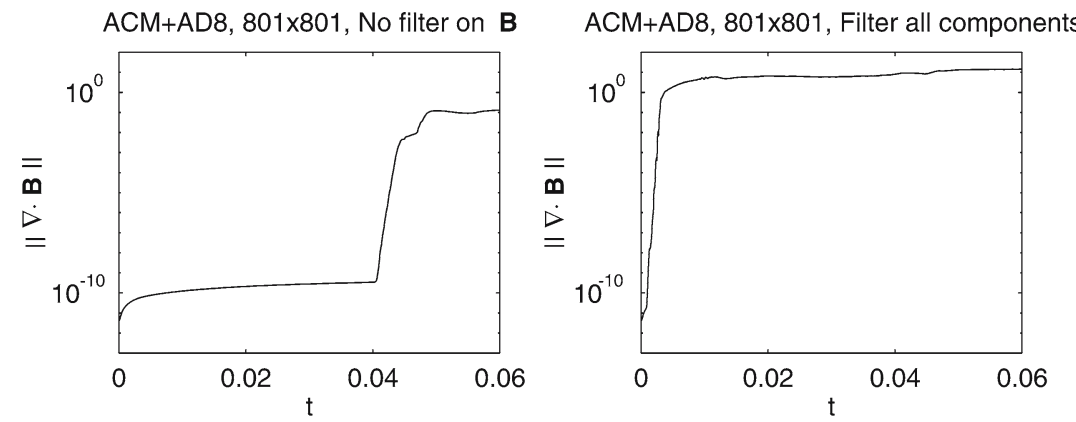

ACM66+AD8 801x801, no filter on B
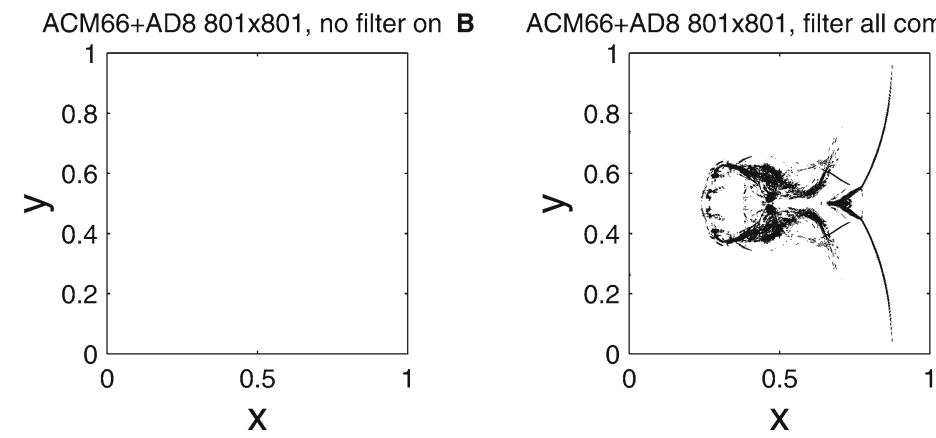

Fig. 19. $L^{2}$-norm of $\nabla \cdot \mathbf{B}$ versus time (top row) and $\nabla \cdot \mathbf{B}$ contours at $T=0.06$ (bottom row) by ACM66+AD8 $(\mathrm{d}=0.001)$ for the shock/cloud problem with filter all (right) and no filter on $\mathbf{B}$ (left) using a $801 \times 801$ grid.

of the $\nabla \cdot \mathbf{B}$ numerical error was proposed and validated using four 2D compressible MHD test cases. The new method of defining high-resolution shock-capturing schemes for the conservative MHD equations is further validated over the preliminary study in [26]. Five levels of grid refinement on four different flow types were compared with three standard high-resolution shock-capturing schemes, namely, a second-order MUSCL and Harten-Yee upwind TVD schemes, and the fifth-order WENO scheme (WENO5). The new method of using the non-conservative eigensystem when solving the conservative equations is also applicable in the context of commonly used shock-capturing schemes for the MHD equations.

The "no filter on B" by the two filter schemes works well for both the conservative and non-conservative systems and exhibits smaller $\nabla \cdot \mathbf{B}$ numerical error than standard shock-capturing methods without traditional divergence cleanings. For periodic boundary conditions and for open boundaries without complex wave interactions near the physical boundaries, these filter schemes are divergence free. In general, for coarse 

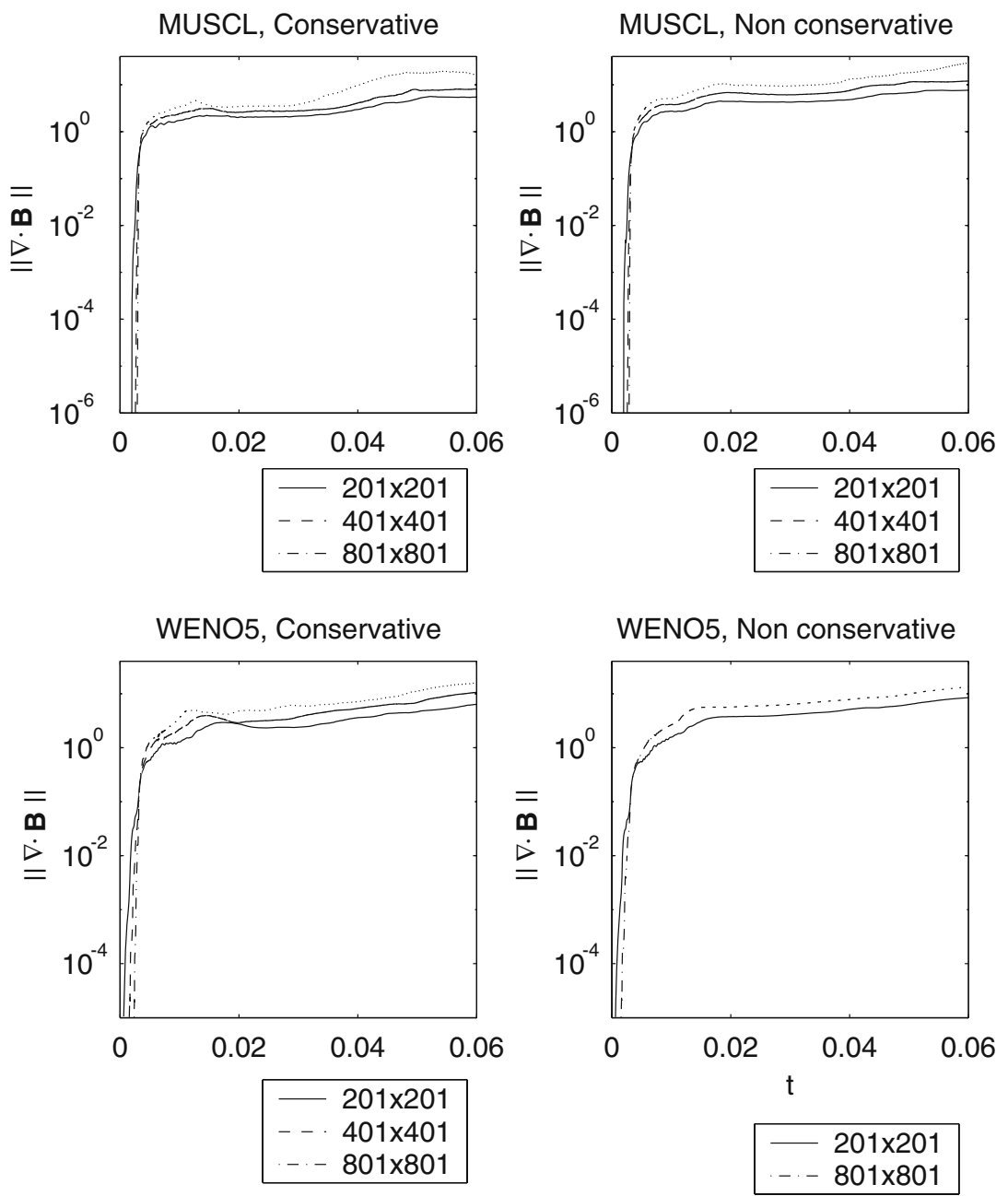

Fig. 20. $L^{2}$-norm of $\nabla \cdot \mathbf{B}$ of MUSCL (top), and WENO5 (bottom) for the shock/cloud problem, conservative (left), non-conservative (right).

grids, the high order methods are more accurate (gas dynamics variables) and require only half the grid points than required by second-order methods. For fine enough grids, in most test cases, the accuracy (gas dynamics variables) is similar for all five methods.

Over all, ACM66 and WAV66 are less stable than ACM66+AD8 and WAV66+AD8. In some cases, ACM66+AD8 is more stable than WAV66+AD8. However, divergence free can be obtained by WAV66+AD8 

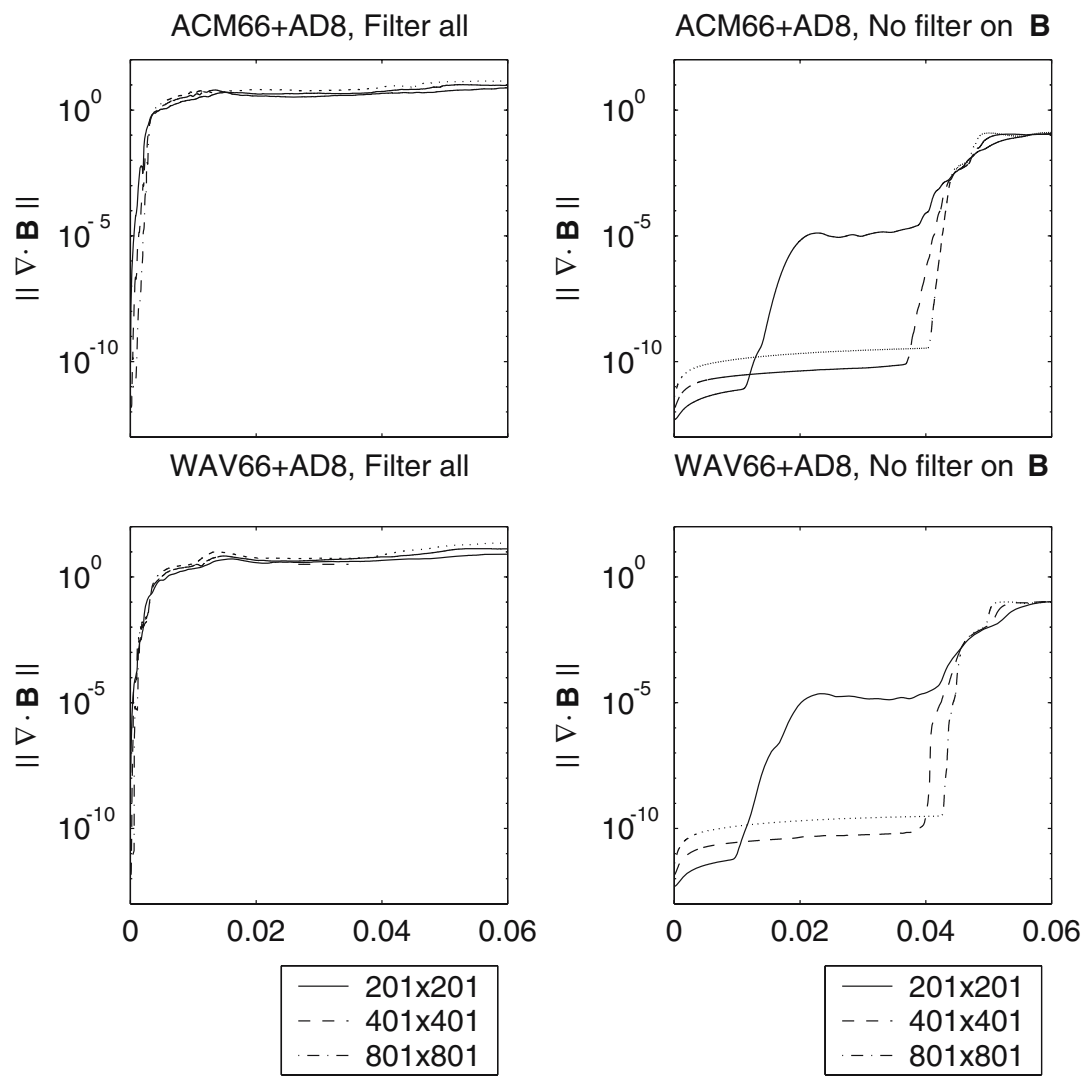

Fig. 21. $L^{2}$-norm of $\nabla \cdot \mathbf{B}$ of ACM66+AD8, $d=0.001$, (top) and WAV66+AD8, $d=0.001$, (bottom), for the shock/cloud problem, filter all (left), no filter B (right).

using the filter all option at an early stage of time development for the Orszag-Tang test case but not by ACM66+AD8. This is due to the fact that the wavelet sensor is capable in detecting very smooth flow and turns off the non-linear filter completely whereas the ACM sensor only reduces the strength at the same region.

The role that the proper treatment of the corresponding numerical boundary conditions can play on the effect of reducing the $\nabla \cdot \mathbf{B}$ numerical error was studied. It was shown that a divergence-free numerical boundary condition plays an important role for a completely divergence-free scheme. There is an added potential complication in a need to employ stable numerical boundary treatments and the requirement of divergence free for spatially higher than second-order centered base schemes. Stable numerical 
boundary conditions for higher than second-order centered schemes for systems of hyperbolic conservation laws were only fully developed in the 1990s using the discrete $L^{2}$ energy norm, sometimes referred to as the SBP conditions. The grid stencil of these SBP boundary operators is very wide and might not be suitable for general multi-dimensional open boundaries with very complex wave interactions. An example of this type of flow is the shock/cloud problem. One difficulty with the SBP boundary operator is that the SBP condition can be easily destroyed when a Neumann or Robin type of physical boundary condition is encountered. Several ways to overcome the difficulty have been suggested by Olsson $[15,16]$ for conservation laws without constraint. For the MHD with an initial condition constraint of $\nabla \cdot \mathbf{B}=0$, we have identified the additional difficulty of how to conserve the $\nabla \cdot \mathbf{B}$ condition at the boundaries without destroying the advantage of the SBP condition. Research in this direction is ongoing.

For all four test cases, MUSCL and Harten-Yee require similar CPU time. The ACM and WAV-filter schemes require slightly more CPU time than the Harten-Yee and MUSCL schemes. For almost all problems, WENO5 requires more CPU time than ACM and WAV-filter schemes. This is due to the fact that both filter schemes require only one Riemann solve/per time step per direction (independent of the time discretizations of the base scheme step) as oppose to two Riemann solves/per time step per direction by the MUSCL, Harten-Yee schemes using a second-order Runge-Kutta method. In addition, for all test cases and all five methods (except the no filter on $\mathbf{B}$ option for the two filter schemes), the $\nabla \cdot \mathbf{B}$ contour numerical errors (at their corresponding stopping times) increase as the grid is refined. For a more detailed comparison and the performance of all five schemes for all the test cases (see [36]). Although this paper concentrates on using the Harten-Yee dissipative portion as part of the non-linear filter, comparison among three different non-linear dissipations (Harten-Yee, MUSCL and WENO5) as part of the non-linear filter is reported in [37].

One shortcoming of the base scheme step of the filter scheme is that it is not completely divergence free for non-uniform grids and general curvilinear grids. However, if the grid aspect ratio on non-uniform grids is small (e.g., within 10\% of unity), numerical experiment on a mixing layer computation indicated that divergence free is still possible for most of the time evolution when the solution is still smooth. After shocks/shears have formed, complete divergence free is not possible. The resulting $\nabla \cdot \mathbf{B}$ numerical error is many orders of magnitude smaller than the "Filter all" option and the three standard shock-capturing schemes. Thus, the minimization of the $\nabla \cdot \mathbf{B}$ numerical error is also possible by the filter approach if low grid aspect ratios are used in curvilinear grids. Application of these 
schemes to viscous non-ideal MHD flows for the generalized Ohm's law with multiscale structure is forthcoming. Preliminary study shows that divergence free is also possible for viscous MHD with resistivity [37, 38].

\section{APPENDIX A: AVERAGE STATES FORMULAS FOR THE 3-D NON-CONSERVATIVE MHD SYSTEM}

Consider the 3-D symmetrizable non-conservative MHD system (2) in the coordinate direction $\mathbf{k}=\left(k_{1} k_{2} k_{3}\right)$ with the velocity vector $\left(u_{1}, u_{2}, u_{3}\right)^{T}$ $=(u, v, w)^{T}$ and the magnetic field vector $\left(B_{1}, B_{2}, B_{3}\right)^{T}=\left(B_{x}, B_{y}, B_{z}\right)^{T}$. Let the right and left given states of the conservative vector be, $U_{L}$ and $U_{R}$, respectively. The Gallice average state for the eight variables $\mathbf{q}=$ $\left(\begin{array}{lllllllll}\rho & u_{1} & u_{2} & u_{3} & h^{*} & B_{1} & B_{2} & B_{3}\end{array}\right)$ denoted by

$$
\overline{\mathbf{q}}=\left(\underline{\rho} \bar{u}_{1} \bar{u}_{2} \bar{u}_{3} \bar{h}^{*} \underline{B}_{1} \underline{B}_{2} \underline{B}_{3}\right),
$$

where the averages are computed as

$$
\begin{array}{ll}
\underline{\rho}=\sqrt{\rho_{L} \rho_{R}}, \quad \bar{u}_{1}=\frac{\left(u_{1}\right)_{L} \sqrt{\rho_{L}}+\left(u_{1}\right)_{R} \sqrt{\rho_{R}}}{\sqrt{\rho_{L}}+\sqrt{\rho_{R}}}, \\
\bar{h}^{*}=\frac{h_{L}^{*} \sqrt{\rho_{L}}+h_{R}^{*} \sqrt{\rho_{R}}}{\sqrt{\rho_{L}}+\sqrt{\rho_{R}}}, \quad \underline{B}_{1}=\frac{\left(B_{1}\right)_{L} \sqrt{\rho_{R}}+\left(B_{1}\right)_{R} \sqrt{\rho_{L}}}{\sqrt{\rho_{L}}+\sqrt{\rho_{R}}}
\end{array}
$$

and similarly for $\bar{u}_{2}, \bar{u}_{3}, \underline{B}_{2}, \underline{B}_{3}$. The modified enthalpy is defined as

$$
h^{*}=\left(e+p+|\mathbf{B}|^{2} / 2\right) / \rho .
$$

Define

$$
X=\left[\left(\left(\Delta B_{1}\right)^{2}+\left(\Delta B_{2}\right)^{2}+\left(\Delta B_{3}\right)^{2}\right) / 2\left(\rho_{L}+\rho_{R}\right)\right]
$$

with the notation

$$
\Delta\left(B_{1}\right)=\left(B_{1}\right)_{R}-\left(B_{1}\right)_{L} .
$$

Similarly, for $\Delta\left(B_{2}\right)$ and $\Delta\left(B_{3}\right)$ and same notation convention will be followed. The average state sound speed squared becomes

$$
c^{2}=(2-\gamma) X+(\gamma-1)\left[\bar{h}^{*}-\frac{1}{2}\left(\bar{u}_{1}^{2}+\bar{u}_{2}^{2}+\bar{u}_{3}^{2}\right)-\left(\underline{B}_{1}^{2}+\underline{B}_{2}^{2}+\underline{B}_{3}^{2}\right) / \underline{\rho}\right]
$$

where the values of the variables are taken from the average state vector $\overline{\mathbf{q}}$. 
The average state fast, Alfven and slow speeds $c_{f}, c_{a}, c_{s}$ can now be computed from the average state and the sound speed (9), according to the formulas

$$
c_{f, s}^{2}=\frac{1}{2}\left[c^{2}+|\underline{\mathbf{B}}|^{2} / \underline{\rho} \pm \sqrt{\left(c^{2}+|\underline{\mathbf{B}}|^{2} / \underline{\rho}\right)^{2}-4 c^{2} \underline{B}^{2} /|\mathbf{k}|^{2} \underline{\rho}}\right]
$$

and

$$
c_{a}=|\underline{B}| /(|\mathbf{k}| \sqrt{\underline{\rho}}),
$$

where the notation $\underline{B}=k_{1} \underline{B}_{1}+k_{2} \underline{B}_{2}+k_{3} \underline{B}_{3}$ and $|\underline{\mathbf{B}}|^{2}=\left(\underline{B}_{1}\right)^{2}+\left(\underline{B}_{2}\right)^{2}+$ $\left(\underline{B}_{3}\right)^{2}$ are used. The average state eigenvalues of the symmetrizable nonconservative MHD system are (subscribe index in increasing order of the eigenvalues)

$$
\lambda_{8,1}=\bar{u} \pm|\mathbf{k}| c_{f}, \quad \lambda_{7,2}=\bar{u} \pm|\mathbf{k}| c_{a}, \quad \lambda_{6,3}=\bar{u} \pm|\mathbf{k}| c_{s}, \quad \lambda_{4,5}=\bar{u},
$$

where we denote $\bar{u}=k_{1} \bar{u}_{1}+k_{2} \bar{u}_{2}+k_{3} \bar{u}_{3}$. For the average state eigenvectors, we compute the magnetic field orthogonal to $\mathbf{k}$,

$$
\mathbf{B}_{\perp}=\underline{\mathbf{B}}-\mathbf{k}^{T} \underline{\mathbf{B}} \frac{\mathbf{k}}{|\mathbf{k}|^{2}},
$$

where $\mathbf{B}$ is the average state magnetic field in $\overline{\mathbf{q}}$. Compute the normalized vector $\mathbf{b}_{\perp}=\mathbf{B}_{\perp} /\left|\mathbf{B}_{\perp}\right|$, orthogonal to $\mathbf{k}$. If $\mathbf{B}_{\perp}$ is zero, define $\mathbf{b}_{\perp}$ as any unit vector $\left(n_{1}, n_{2}, n_{3}\right)$ which is orthogonal to $\mathbf{k}$. The eigenvectors are given as

$$
\begin{aligned}
& \mathbf{r}_{8,1}=\left(\begin{array}{c}
\rho \alpha_{f} \\
\underline{\rho}\left(\alpha_{f}\left(\bar{u}_{1} \pm c n_{1}\right) \mp \alpha_{s} c_{s} s b_{\perp, 1}\right) \\
\underline{\rho}\left(\alpha_{f}\left(\bar{u}_{2} \pm c_{f} n_{2}\right) \mp \alpha_{s} c_{s} s b_{\perp, 2}\right) \\
\underline{\rho}\left(\alpha_{f}\left(\bar{u}_{3} \pm c_{f} n_{3}\right) \mp \alpha_{s} c_{s} s b_{\perp, 3}\right) \\
\underline{\rho} \alpha_{f}\left(\bar{h}^{*} \pm \tilde{u} c_{f}-|\underline{\mathbf{B}}|^{2} / \rho\right) \mp \rho \alpha_{s} c_{s} s\left(\overline{\mathbf{u}}^{T} \mathbf{b}_{\perp}\right)+\sqrt{\underline{\rho}} \alpha_{s} c\left|\mathbf{B}_{\perp}\right| \\
\bar{\alpha}_{s} \sqrt{\bar{\rho}} c b_{\perp, 1} \\
\alpha_{s} \sqrt{\bar{\rho}} c b_{\perp, 2} \\
\alpha_{s} \sqrt{\underline{\rho}} c b_{\perp, 3}
\end{array}\right), \\
& \mathbf{r}_{7,2}=\left(\begin{array}{c}
0 \\
\pm \rho\left(\mathbf{b}_{\perp} \times \mathbf{k}\right)_{1} \\
\pm \bar{\rho}\left(\mathbf{b}_{\perp} \times \mathbf{k}\right)_{2} \\
\pm \bar{\rho}\left(\mathbf{b}_{\perp} \times \mathbf{k}\right)_{3} \\
\left. \pm \rho \overline{\overline{\mathbf{u}}} T\left(\mathbf{b}_{\perp} \times \mathbf{k}\right)\right] \\
-\bar{s} \sqrt{\underline{\rho}}\left(\mathbf{b}_{\perp} \times \mathbf{k}\right)_{1} \\
-s \sqrt{\underline{\rho}}\left(\mathbf{b}_{\perp} \times \mathbf{k}\right)_{2} \\
-s \sqrt{\bar{\rho}}\left(\mathbf{b}_{\perp} \times \mathbf{k}\right)_{3}
\end{array}\right)
\end{aligned}
$$




$$
\mathbf{r}_{6,3}=\left(\begin{array}{c}
\underline{\rho} \alpha_{s} \\
\underline{\rho}\left(\alpha_{s}\left(\bar{u}_{1} \pm c_{s} n_{1} \pm \alpha_{f} c_{f} s b_{\perp, 1}\right)\right. \\
\underline{\rho}\left(\alpha_{s}\left(\bar{u}_{2} \pm c_{s} n_{2}\right) \pm \alpha_{f} c_{f} s b_{\perp, 2}\right) \\
\underline{\rho}\left(\alpha_{s}\left(\bar{u}_{3} \pm c_{s} n_{3} \pm \alpha_{f} c_{f} s b_{\perp, 3}\right)\right. \\
\underline{\rho} \alpha_{s}\left(\bar{h}^{*} \pm \tilde{u} c_{f}-|\underline{\mathbf{B}}|^{2} / \underline{\rho} \pm \underline{\rho} \alpha_{f} c_{f} s\left(\overline{\mathbf{u}}^{T} \mathbf{b}_{\perp}\right)-\sqrt{\underline{\rho}} \alpha_{f} c\left|\mathbf{B}_{\perp}\right|\right. \\
-\alpha_{f} \sqrt{\underline{\rho}} c b_{\perp, 1} \\
-\alpha_{f} \sqrt{\underline{\rho}} c b_{\perp, 2} \\
-\alpha_{f} \sqrt{\bar{\rho}} c b_{\perp, 3}
\end{array}\right)
$$

and

$$
\mathbf{r}_{4}=\left(\begin{array}{c}
1 \\
\bar{u}_{1} \\
\bar{u}_{2} \\
\bar{u}_{3}\left(\bar{u}_{1}^{2}+\bar{u}_{2}^{2}+\bar{u}_{3}^{2}\right)+\frac{\gamma-2}{\gamma-1} X \\
0 \\
0 \\
0
\end{array}\right), \quad \mathbf{r}_{5}=\left(\begin{array}{c}
0 \\
0 \\
0 \\
0 \\
\frac{B}{k_{1}} \\
k_{2} \\
k_{3}
\end{array}\right)
$$

In the eigenvectors above, all variables (except $X$ ) as well as the quantities

$$
\begin{aligned}
& n_{1}=k_{1} /|\mathbf{k}|, \quad n_{2}=k_{2} /|\mathbf{k}|, \quad n_{3}=k_{3} /|\mathbf{k}|, \quad \tilde{u}=\bar{u} /|\mathbf{k}| \\
& s=\operatorname{sign} \underline{B}, \quad \alpha_{f}^{2}=\left(c^{2}-c_{s}^{2}\right) /\left(c_{f}^{2}-c_{s}^{2}\right), \quad \alpha_{s}^{2}=\left(c_{f}^{2}-c^{2}\right) /\left(c_{f}^{2}-c_{s}^{2}\right)
\end{aligned}
$$

are computed at the average state $\overline{\mathbf{q}}$, or through the wave speeds derived from $\overline{\mathbf{q}}$ as described in (9), (10), and (11).

For all the high-resolution shock-capturing schemes as well as the filter schemes which involved the use of the Roe-type approximate solver (dimension-by-dimension), the jump in the local characteristic variables $\bar{\alpha}$ are needed. Here, $\bar{\alpha}$ has the form

$$
\bar{\alpha}=\bar{R}^{-1}\left(U_{R}-U_{L}\right)=\bar{R}^{-1} \Delta U
$$

where $\bar{R}^{-1}$ is the inverse of the average state of the above eigenvectors.

Instead of obtaining the matrix $\bar{R}^{-1}$ and performing the matrix and vector multiplications at every grid points, the formulas for $\bar{\alpha}$ given below 
are more economic to compute. Define first

$$
\begin{aligned}
& a_{1 p}=\frac{1}{\rho c^{2}}\left[\alpha_{f} \Delta p+\alpha_{f} X \Delta \rho+\sqrt{\underline{\rho}} c \alpha_{s}\left(\mathbf{b}_{\perp}^{T} \Delta \mathbf{B}\right)\right], \\
& a_{1 m}=\frac{1}{c^{2}}\left(-s \alpha_{s} c_{s}\left(\mathbf{b}_{\perp}^{T} \Delta \mathbf{u}\right)+\alpha_{f} c_{f}\left(\mathbf{n}^{T} \Delta \mathbf{u}\right)\right), \\
& a_{2 p}=\frac{s}{\sqrt{\underline{\rho}}|\mathbf{k}|^{2}} \mathbf{k}^{T}\left(\mathbf{b}_{\perp} \times \Delta \mathbf{B}\right), \\
& a_{2 m}=-\frac{1}{|\mathbf{k}|^{2}} \mathbf{k}^{T}\left(\mathbf{b}_{\perp} \times \Delta \mathbf{u}\right), \\
& a_{3 p}=\frac{1}{\frac{\rho}{\rho} c^{2}}\left(\alpha_{s} \Delta p+\alpha_{s} X \Delta \rho-\sqrt{\underline{\rho}} c \alpha_{f}\left(\mathbf{b}_{\perp}^{T} \Delta \mathbf{B}\right)\right), \\
& a_{3 m}=\frac{1}{c^{2}}\left(s \alpha_{f} c_{f}\left(\mathbf{b}_{\perp}^{T} \Delta \mathbf{u}\right)+\alpha_{s} c_{s}\left(\mathbf{n}^{T} \Delta \mathbf{u}\right)\right) .
\end{aligned}
$$

The $l$ th element of $\bar{\alpha}$ (denoted by $\bar{\alpha}^{l}$ ) are then given by

$$
\begin{aligned}
& \bar{\alpha}^{1}=a_{1 p}-a_{1 m}, \quad \bar{\alpha}^{2}=a_{2 p}-a_{2 m}, \quad \bar{\alpha}^{3}=a_{3 p}-a_{3 m}, \\
& \bar{\alpha}^{4}=\left(\left(c^{2}-X\right) \Delta \rho-\Delta p\right) / c^{2}, \quad \bar{\alpha}^{5}=\mathbf{k}^{T} \Delta \mathbf{B} /|\mathbf{k}|^{2}, \\
& \bar{\alpha}^{6}=a_{3 p}+a_{3 m}, \quad \bar{\alpha}^{7}=a_{2 p}+a_{2 m}, \quad \bar{\alpha}^{8}=a_{1 p}+a_{1 m} .
\end{aligned}
$$

For high-resolution shock-capturing schemes involving the use of flux limiters on the elements of $\bar{\alpha}$ in simulating high speed flows when the jump of the pressure is more pronounced than the jump in the energy, it is advantageous to scale the eigenvectors so that $\bar{\alpha}$ is proportional to the jump in the pressure instead of the energy (see [31]). We suggest using the eigenvectors $\mathbf{r}^{\prime}$ instead, where

$$
\mathbf{r}_{1,3,6,8}^{\prime}=\mathbf{r}_{1,3,6,8} /\left(\underline{\rho} c^{2}\right), \quad \mathbf{r}_{2,7}^{\prime}=\mathbf{r}_{2,7} /(\underline{\rho} c), \quad \mathbf{r}_{4}^{\prime}=\mathbf{r}_{4} / c^{2}, \quad \mathbf{r}_{5}^{\prime}=\mathbf{r}_{5} /(\sqrt{ } \underline{\rho} c) .
$$

The correspondingly $\bar{\alpha}$ should be scale accordingly (i.e., scale by the inverse of what is used for the eigenvectors).

To this end, when applying the above average state at, for example, the average state grid index $(j+1 / 2, k, l)$, the right state $U_{R}$ should be replaced by $U_{j+1, k, l}$, and the left state $U_{L}$ should be replaced by $U_{j, k, l}$. The element $\bar{\alpha}^{l}$ now becomes $\alpha_{j+1 / 2, k, l}^{l}\left(\alpha_{j+1 / 2}^{l}\right)$ and the rest of the average state eigenvalues and eigenvectors accordingly.

\section{APPENDIX B}

This appendix shows the non-linear dissipative portion of the Harten-Yee scheme, the three flux (slope) limiters, the form of the ACM sensor and 
a summary of the wavelet sensor that was used to solve the four test cases. A comparison of using three different dissipative portions of typical shock-capturing schemes (Harten-Yee, MUSCL, and WENO5) is reported in [38].

\section{Non-Linear Dissipative Portion of the Harten-Yee Scheme}

The Harten-Yee scheme (for an one-time level time discretization) can be written as

$$
\begin{aligned}
U_{j, k, l}^{n+1}= & U_{j, k, l}^{n}-\frac{\Delta t}{\Delta x}\left[\widetilde{F}_{j+1 / 2}^{n}-\widetilde{F}_{j-1 / 2}^{n}\right]-\frac{\Delta t}{\Delta y}\left[\widetilde{H}_{k+1 / 2}^{n}-\widetilde{H}_{k-1 / 2}^{n}\right] \\
& -\frac{\Delta t}{\Delta z}\left[\widetilde{G}_{l+1 / 2}^{n}-\widetilde{G}_{l-1 / 2}^{n}\right] .
\end{aligned}
$$

The $x$-numerical flux $\widetilde{F}_{j+1 / 2}$ has the form

$$
\widetilde{F}_{j+1 / 2}=\frac{1}{2}\left[F_{j+1, k, l}+F_{j, k, l}+R_{j+1 / 2} \Phi_{j+1 / 2}\right] .
$$

The non-linear dissipative portions of the Harten-Yee scheme are $\Phi_{j+1 / 2}$ and $\Phi_{j-1 / 2}$. For all the numerical experiments, the non-linear dissipative portions of the Harten-Yee scheme are used as part of the non-linear filter. Denote the $l$ th-element of $\Phi_{j+1 / 2}$ as $\phi_{j+1 / 2}^{l}$. In this case, the $\phi_{j+1 / 2}^{l}$ in (8) for the $j$-direction is

$$
\begin{gathered}
\phi_{j+1 / 2}^{l}=\frac{1}{2} Q\left(a_{j+1 / 2}^{l}\right)\left[g_{j+1}^{l}+g_{j}^{l}\right]-Q\left(a_{j+1 / 2}^{l}+\gamma_{j+1 / 2}^{l}\right) \alpha_{j+1 / 2}^{l}, \\
\gamma_{j+1 / 2}=\frac{1}{2} Q\left(a_{j+1 / 2}^{l}\right) \begin{cases}\left(g_{j+1}^{l}-g_{j}^{l}\right) / \alpha_{j+1 / 2}^{l}, & \alpha_{j+1 / 2}^{l} \neq 0, \\
0, & \alpha_{j+1 / 2}^{l}=0\end{cases}
\end{gathered}
$$

with $Q(x)=\sqrt{x^{2}+\epsilon^{2}}$, the entropy satisfying remedy for the scheme with entropy correction parameter $\epsilon \cdot a_{j+1 / 2}^{l}$ is the $l$ th characteristic speed, $\gamma_{j+1 / 2}^{l}$ is the modified characteristic speed and $g_{j}^{l}$ is a slope limiter which is a function of $\alpha_{j \pm 1 / 2}^{l}$, the jump in the characteristic variable in the $x$-direction. Here $\alpha_{j \pm 1 / 2}^{l}$ are the $l$ th elements of $R_{j \pm 1 / 2}^{-1}\left(U_{j+1}-U_{j}\right)$. All the variables with the subscripts " $j+1 / 2$ " are evaluated at the Gallice average state in the $j$-direction (see Appendix A for the average state formula).

\section{Flux or Slope Limiters}

The three limiters used for the four test cases are the minmod, van Albada and Colella and Woodward limiters. They are of the following 
form, respectively

$$
\begin{aligned}
g_{j}^{l}= & \operatorname{minmod}\left(\alpha_{j-\frac{1}{2}}^{l}, \alpha_{j+\frac{1}{2}}^{l}\right), \\
g_{j}^{l}= & \left\{\alpha_{j-\frac{1}{2}}^{l}\left[\left(\alpha_{j+\frac{1}{2}}^{l}\right)^{2}+\delta_{2}\right]+\alpha_{j+\frac{1}{2}}^{l}\left[\left(\alpha_{j-\frac{1}{2}}^{l}\right)^{2}+\delta_{2}\right]\right\} / \\
& {\left[\left(\alpha_{j+\frac{1}{2}}^{l}\right)^{2}+\left(\alpha_{j-\frac{1}{2}}^{l}\right)^{2}+2 \delta_{2}\right] } \\
g_{j}^{l}= & \operatorname{minmod}\left(2 \alpha_{j-\frac{1}{2}}^{l}, 2 \alpha_{j+\frac{1}{2}}^{l}, \frac{1}{2}\left(\alpha_{j+\frac{1}{2}}^{l}+\alpha_{j-\frac{1}{2}}^{l}\right)\right) .
\end{aligned}
$$

Here $\delta_{2}$ is a small dimensionless parameter to prevent division by zero. In practical calculations $10^{-7} \leq \delta_{2} \leq 10^{-5}$ is a commonly used range. The minmod function of a list of arguments is equal to the smallest number in absolute value if the list of arguments is of the same sign, or is equal to zero if any arguments are of opposite sign. Note that the same form of the limiters for the MUSCL scheme is used except $\alpha_{j+1 / 2}^{l}$ will be replaced by the jump in the primitive variables if primitive variables are used for the application of the slope limiters.

Next, we discuss the ACM and wavelet sensors. These sensors are scheme independent and are not restricted to use with the non-linear dissipative portion of the Harten-Yee scheme.

\section{ACM AND Wavelet Sensors}

A form of the ACM sensor $\left(s^{N}\right)_{j+1 / 2}^{l}$ proposed in [32] is

$$
\left(s^{N}\right)_{j+1 / 2}^{l}=\kappa \max \left(\theta_{j}^{l}, \theta_{j+1}^{l}\right)
$$

where

$$
\theta_{j}=\left|\frac{\left|\alpha_{j+1 / 2}^{l}\right|-\left|\alpha_{j-1 / 2}^{l}\right|}{\left|\alpha_{j+1 / 2}^{l}\right|+\left|\alpha_{j-1 / 2}^{l}\right|}\right|
$$

The parameter $\kappa$ is in the range of $(1,0.5)$ for each wave. For example, $\kappa$ is larger for non-linear waves and $\kappa$ is smaller for the degenerate wave (see [32, 33] for details).

It was shown in [22] that the method can be improved by letting the sensor $\left(s^{N}\right)_{j+1 / 2}^{l}$ be based instead on a regularity estimate obtained from the wavelet coefficients of the flow solution. The wavelet analysis gives an estimate of the so-called local Lipschitz exponent $\beta$ at each grid cell. The dissipation is switched on for low $\beta$ values, and switched off when $\beta$ 
becomes large [22]. The wavelet analysis is more general and can be used to detect other features besides shocks/contacts. In addition, the wavelet sensor can be used as an improved indicator over commonly used detectors for grid adaptation.

\section{Wavelet Sensor for Multiscale Flow Physics}

The CPU time to compute the ACM sensor is nearly the same as the wavelet sensor. However, one needs some background in multi-resolution wavelets. It is too lengthy to be repeated here. Interested reader should refer to $[23,34]$ for details. Here, the basic idea for obtaining the wavelet in descriptive form is summarized.

(I) Wavelet type: (non-orthogonal multi-resolution wavelets)

- redundant form of Harten's multi-resolution form,

- second-order B-splines,

- wavelets that can distinguish spurious high frequency oscillations from turbulence.

(II) Flow variables to be sensed

- density and pressure,

- local characteristic variables,

- primitive variables,

- entropy variables.

(III) Procedures

- apply wavelets to the flow variable vector to be sensed,

- obtain the corresponding wavelet coefficients at each grid point (usually involves 2-4 levels of nested difference operators),

- obtain the corresponding Lipschitz exponent $\beta_{j}$ of the wavelet coefficients (e.g., least square fit of the wavelet coefficients in domain of dependence),

- determine the cut off Lipschitz exponent $\left(s^{N}\right)_{j+1 / 2}^{l}$ at each grid point. In this case $\left(s^{N}\right)_{j+1 / 2}^{l}$ consists of either " 0 "s or " 1 "s. An alternative is to have a smooth transition between 0 and 1),

- use Lipschitz exponent cut off values $\left(s^{N}\right)_{j+1 / 2}^{l}$ as indicators to switch on or off the appropriate numerical dissipations at each grid point,

- the same $\left(s^{N}\right)_{j+1 / 2}^{l}$ sensor can be used as indicators for grid adaption if desired. 


\section{REFERENCES}

1. Brackbill, J. U., and Barnes, D. C. (1980). Note: The effect of nonzero $\nabla \cdot B$ on the numerical solution of the magnetohydrodynamic equations. J. Comput. Phys. 35, 426430.

2. Brio, M., and Wu, C. C. (1998). An upwind differencing scheme for the equations of ideal magnetohydrodynamics. J. Comput. Phys. 75, 400-422.

3. Cargo, P., and Gallice, G. (1997). Roe matrices for ideal MHD and systematic construction of Roe matrices for systems of conservation laws. J. Comput. Phys. 136, 446-466.

4. Dedner, A., Kemm, F., Kröner, D., Munz, C.-D., Schnitzer, T., and Wesenberg, M. (2002). Hyperbolic divergence cleaning for the MHD equations. J. Comput. Phys. 175, 645-673.

5. Dai, W., and Woodward, P. R. (1998). A simple finite difference scheme for multidimensional magnetohydrodynamical equations. J. Comput. Phys. 142, 331-369.

6. Dai, W., and Woodward, P. R. (1998). On the divergence-free condition and conservation laws in numerical simulations for supersonic magnetohydrodynamic flows. Astrophys. $J$. 494, 317-335.

7. De Sterck, H. (2001). Multi-dimensional upwind constrained transport on unstructured grids for shallow water magnetohydrodynamics. AIAA Paper 2001-2623.

8. Evans, C. R., and Hawley, J. F. (1988). Simulation of magnetohydrodynamic flows: a constrained transport method. Astrophys. J. 332, 659-677.

9. Gallice, G. (1997). Systéme dÉuler-Poisson, Magnétohydrodynamique et Schemeas de Roe, $\mathrm{PhD}$ Thesis, L'Université Bordeaux I.

10. Gallice, G. Resolution numerique des equations de la magnetohydrodynamique ideale bidimensionnelle. Actes du workshop Méthodes Numériques pour la M.H.D., CIRM (Luminy), December 4-5, 1995.

11. Godunov, S. K. (1972). Symmetric form of the equations of magnetohydrodynamics. Num. Methods Mech Continuum Medium 13(1), 26-34.

12. Harten, A., and Hyman, J. M. (1983). A self-adjusting grid for the computation of weak solutions of hyperbolic conservation laws. J. Comput. Phys. 50, 235-269.

13. Jiang, G.-S., and Shu, C.-W. (1996). Efficient implementation of weighted ENO schemes. J. Comput. Phys. 126, 202-228.

14. Nordstrom, J., and Carpenter, M. H. (1999). Boundary and interface conditions for highorder finite-difference schemes applied to the Euler and Navier-Stokes equations. J. Comput. Phys. 148, 621-645.

15. Olsson, P. (1995). Summation by parts, projections and stability, I. Math. Comp. 64, 1035-1065.

16. Olsson, P. (1995). Summation by parts, projections and stability. III. RIACS Technical Report 95-06, NASA Ames Research Center.

17. Olsson P., and Oliger, J. (1994). Energy and maximum norm estimates for nonlinear conservation laws. RIACS Technical Report 94.01.

18. Orszag, S. A., and Tang, C. M. (1979). Small-scale structure of two-dimensional magnetohydrodynamic turbulence. J. Fluid Mech. 90, 129-143.

19. Powell, K. G. (1994). An approximate Riemann solver for magnetohydrodynamics (that works in more than one dimension). ICASE-Report 94-24, NASA Langley Research Center, April 1994.

20. Powell, K. G., Roe, P. L., Linde, T. J., Gombosi, T. I., and De Zeeuw, D. L. (1999). A solution-adaptive upwind scheme for ideal magnetohydrodynamics. J. Comput. Phys. 154, 284-309. 
21. Sjögreen, B. (1995). High order centered difference methods for the compressible NavierStokes equations. J. Comput. Phys. 117, 67-78.

22. Sjögreen, B., and Yee, H. C. (2004). Multiresolution wavelet based adaptive numerical dissipation control for shock-turbulence computation. RIACS Technical Report TR01.01, NASA Ames research center (Oct 2000); also, J. Sci. Comput. 20, 211-255.

23. Sjögreen, B., and Yee, H. C. (2003). Grid convergence of high order methods for multiscale complex unsteady viscous compressible flows. RIACS Technical Report TR01.06, April, 2001, NASA Ames research center; AIAA 2001-2599. In Proceedings of the 15th AIAA CFD Conference, June 11-14, 2001, Anaheim, CA., also, J. Comput. Phys. 185, 126.

24. Sjögreen, B., and Yee, H. C. (2003). Low dissipative high order numerical simulations of supersonic reactive flows. RIACS Technical Report TR01-017, NASA Ames Research Center (May 2001); In Proceedings of the ECCOMAS Computational Fluid Dynamics Conference 2001, Swansea, Wales, UK, September 4-7, 2001; also, Int. J. Num. Meth. fluids 43, 1221-1238.

25. Sjögreen, B., and Yee, H. C. (2002). Analysis of high order difference methods for multiscale complex compressible flows. In Proceedings of the 9th International Conference on Hyperbolic Problems, March 25-29, Pasadena, CA.

26. Sjögreen, B., and Yee, H. C. (2003). Efficient low dissipative high order schemes for multiscale MHD flows, I: basic theory. AIAA 2003-4118. In Proceedings of the 16th AIAA/CFD Conference, June 23-26, Orlando, Fl.

27. Sjögreen, B., and Yee, H. C. (2005). Efficient low dissipative high order schemes for multiscale MHD flows, III: curvilinear grid simulations. RIACS Technical Report, NASA Ames Research Center.

28. Tóth, G. (2000). The $\operatorname{div} \mathrm{B}=0$ constraint in shock-capturing magnetohydrodynamic codes. J. Comput. Phys. 161, 605-652.

29. Vinokur, M. (1996). A rigorous derivation of the MHD equations based only on Faraday's and Ampére's Laws. Presentation at LANL MHD Workshop on $\nabla \cdot \mathbf{B}$ Cleaning, August.

30. Vinokur, M., and Yee, H. C. (2002). Extension of efficient low dissipative high order schemes for 3-D curvilinear moving grids. NASA TM 209598, June 2000; also, In Frontiers of Computational Fluid Dynamics, World Scientific, Caughey, D. A, and Hafez, M.M.(eds), 129-164.

31. Yee, H. C. (1989). A class of high-resolution explicit and implicit shock-capturing methods. VKI Lecture Series 1989-04, March 6-10, also NASA TM-101088, February 1989.

32. Yee, H. C., Sandham, N. D., and Djomehri, M. J. (1999). Low dissipative high order shock-capturing methods using characteristic-based filters. J. Comput. Phys. 150, 199238.

33. Yee, H. C., Vinokur, M., and Djomehri, M. J. (2000). Entropy splitting and numerical dissipation. J. Comput. Phys. 162, 33-81.

34. Yee, H. C., and Sjögreen, B. (2002). Designing adaptive low dissipative high order schemes for long-time integrations. In Turbulent Flow Computation, Drikakis, D., and Geurts, B., Kluwer Academic Publisher; also RIACS Technical Report TR01-28, December 2001.

35. Yee, H. C., and Sjögreen, B. (2003). Divergence free high order filter methods for the compressible MHD equations. In Proceedings of the International Conference on High Performance Scientific Computing, March 10-14, Hanoi, Vietnam.

36. Yee, H. C., and Sjögreen, B. (2003). Efficient low dissipative high order schemes for multiscale MHD flows, II: minimization of $\nabla$. B numerical error. RIACS Technical Report TR03.10, NASA Ames Research Center. 
37. Yee, H. C., and Sjögreen, B. (2004). Adaptive numerical dissipation control in high order schemes for multi-D non-ideal MHD. In Proceedings of the ICCFD3, July 12-16, Toronto, Canada.

38. Yee, H. C., and Sjögreen, B. (2004). Nonlinear filtering and limiting in high order methods for ideal and Non-ideal MHD. In Proceedings of the ICOSAHOM04, June 21-25, Brown University, Providence, RI.

39. Yee, K. S. (1966). Numerical solution of initial boundary value problems involving Maxwell's equations in isotropic media. IEEE Trans. Antennas Propagat. 14, 302-307.

40. Zachary, A. L., Malagoli, A., and Colella, P. (1994). A higher-order Godunov method for multidimensional ideal magnetohydrodynamics. SIAM J. Sci. Comput. 15, 263-284. 